\title{
Where Infinite Spin Particles are Localizable
}

\author{
Roberto Longo ${ }^{1}$, Vincenzo Morinelli ${ }^{1}$, Karl-Henning Rehren ${ }^{2}$ \\ 1 Dipartimento di Matematica, Università di Roma Tor Vergata, Via della Ricerca Scientifica, 1, 00133 Rome, \\ Italy. E-mail: longo@mat.uniroma2.it; morinell@mat.uniroma2.it \\ 2 Institut für Theoretische Physik, Universität Göttingen, 37077 Göttingen, Germany. \\ E-mail: rehren@theorie.physik.uni-goettingen.de
}

Received: 22 May 2015 / Accepted: 9 July 2015

Published online: 29 October 2015 - () The Author(s) 2015. This article is published with open access at Springerlink.com

\begin{abstract}
Particle states transforming in one of the infinite spin representations of the Poincaré group (as classified by E. Wigner) are consistent with fundamental physical principles, but local fields generating them from the vacuum state cannot exist. While it is known that infinite spin states localized in a spacelike cone are dense in the one-particle space, we show here that the subspace of states localized in any double cone is trivial. This implies that the free field theory associated with infinite spin has no observables localized in bounded regions. In an interacting theory, if the vacuum vector is cyclic for a double cone local algebra, then the theory does not contain infinite spin representations. We also prove that if a Doplicher-Haag-Roberts representation (localized in a double cone) of a local net is covariant under a unitary representation of the Poincaré group containing infinite spin, then it has infinite statistics. These results hold under the natural assumption of the Bisognano-Wichmann property, and we give a counter-example (with continuous particle degeneracy) without this property where the conclusions fail. Our results hold true in any spacetime dimension $s+1$ where infinite spin representations exist, namely $s \geq 2$.
\end{abstract}

\section{Introduction}

The classical notion of particles as pointlike objects is meaningless in quantum mechanics. Here the wave function satisfies the Schrödinger equation and the Heisenberg uncertainty relation prevents a sharp localization; increasing energy is needed for better localization. We are going to discuss the intrinsic particle localization properties, and show why infinite spin particles exhibit an essential difference from finite spin particles in this respect.

Roberto Longo: Supported in part by the ERC Advanced Grant 669240 QUEST "Quantum Algebraic Structures and Models", PRIN-MIUR and GNAMPA-INdAM.

Vincenzo Morinelli: Supported in part by PRIN-MIUR and GNAMPA-INdAM. 
Wigner particles and classification of Poincaré group representations. In relativistic quantum mechanics, one better defines a particle through its symmetry, rather than localization, property. The Schrödinger equation is replaced by the Lorentz invariant Klein-Gordon equation and this point of view led to define a particle as an irreducible, positive energy, projective unitary representation of the Poincaré group $\mathcal{P}_{+}^{\uparrow}$, hence to an irreducible, positive energy, unitary representation of the double (universal) cover of $\mathcal{P}_{+}^{\uparrow}$. These are the "minimal" Poincaré covariant objects, the building blocks of any more complete theory.

Within this point of view, Wigner [34] obtained his famous classification of the irreducible, positive energy, unitary representations of the double cover of $\mathcal{P}_{+}^{\uparrow}$, which is isomorphic to $\mathbb{R}^{4} \rtimes \mathrm{SL}(2, \mathbb{C})$.

We briefly recall that a unitary, positive energy representation $U$ is classified, up to unitary equivalence, by two parameters $m$ and $s$. The mass $m$ takes values in $[0, \infty)$ (the lower point in the energy spectrum). If $m>0$, then the values of the spin $s$ are $0, \frac{1}{2}, 1, \frac{3}{2}, 2, \ldots$ (the unitary representations of the cover of the rotation subgroup).

In the mass zero case, the representations fall into two distinct classes according to the representations of the little group, which is the double cover of $E(2)$, the Euclidean group of the plane. The representation with trivial $E(2)$-translations are representations of the (double) torus, labelled by the helicity, a parameter $s$ that takes the place of the spin, $s=0, \frac{1}{2}, 1, \frac{3}{2}, 2, \ldots$.

The remaining massless representations correspond to infinite-dimensional, irreducible representations of the double cover of $E(2)$ and are labelled by a parameter $\kappa>0$ (the radius of the circle that is the joint spectrum of the $E(2)$-translations) and $\mathrm{a} \pm$ sign (Bose/Fermi alternative). They are called infinite spin (or continuous spin) representations.

Infinite spin particles have so far not been observed in nature, although they are compatible with all physical first principles, and are usually disregarded without further explanation. A result by Yngvason [36] shows that they cannot appear in a Wightman theory [36] since no Wightman fields (which have pointlike localization) transforming under an infinite spin representation can exist. One of the main aims of this paper is to study the peculiar localization property of these particles, so as to explain why they are not observable in finite space and time. They are however localizable in certain unbounded spacetime regions [4] (cf. also [17]). Indeed, the authors of [27] have constructed such fields $\Phi(x, e)$, which are localized along rays $x+\mathbb{R}_{+} \cdot e$, where $e$ is a spacelike direction.

We mention at this point that more general notions of particles are necessary to describe situations where, for example, infrared clouds are present, cf. [6]; these will not be considered in the present paper.

The main body of this article deals with the issue of localization of (one-particle) states. In Sect. 9, we present some consequences for the localization of algebras of observables (in the sense of spacelike commutation relations). The one-particle results directly pass to free fields by second quantization, and we shall discuss general results in the interacting case.

Localized particle states. Given a particle, namely an irreducible, positive energy representation $U$ of $\mathcal{P}_{+}^{\uparrow}$, what are the localized states of $U$ ?

If we restrict our attention to finite spin particles, the answer is well known in the quantum field theory context, where one assumes the existence of a local free field transforming in a given representation. In the scalar case, for simplicity, the one-particle Hilbert space $\mathcal{H}$ can be obtained by equipping the Schwartz function space $S\left(\mathbb{R}^{s+1}\right)$ 
with a scalar product, given by the two-point function $(f, g)=(\Phi(f) \Omega, \Phi(g) \Omega)$ of the field. Its Hilbert space closure $\mathcal{H}$ can be viewed as the space of positive energy solutions to the Klein-Gordon wave equation, and carries an irreducible representation $U$ of $\mathcal{P}_{+}^{\uparrow}$ with zero spin/helicity. The localization of one-particle states is given by the support of the Schwartz functions: by assigning to an open region $X$ the closed real linear subspace $H_{\Phi}(X) \subset \mathcal{H}$, the closure of the space of real smooth functions with support in $X$, one obtains a local $U$-covariant net of standard subspaces of $\mathcal{H}$ (see below). The locality of the field, together with the identity $i \Im(f, g)=\left(\Omega,\left[\Phi(f)^{*}, \Phi(g)\right] \Omega\right)$, imply that two subspaces $H_{\Phi}(X)$ and $H_{\Phi}(Y)$ are symplectically orthogonal whenever $X$ and $Y$ are spacelike separated.

In the sequel, we describe the procedure of modular localization, which intrinsically associates with a given representation the states localized in a region $X$, without referring to a local field.

Terminology. A wedge region $W$ is a Poincare transform of the standard wedge $W_{0}=$ $\left\{x \in \mathbb{R}^{4}: x_{3}>\left|x_{0}\right|\right\}$, and $\mathcal{W}$ is the set of all wedge regions. The standard one-parameter family of boosts preserving $W_{0}$ is called $\Lambda_{W_{0}}(t)$, and we put $\Lambda_{W}(t):=g \Lambda_{W_{0}}(t) g^{-1}$ if $W=g\left(W_{0}\right)$. A double cone $O$ is the open intersection of a future and a backward light cone, and $\mathcal{O}$ is the set of all double cones. A spacelike cone is a region of the form $C=x+\bigcup_{t>0} t \cdot O$ where $x \in \mathbb{R}^{4}$ and $O \in \mathcal{O}$ is a double cone spacelike to the point 0 , and $\mathcal{C}$ is the set of all spacelike cones. Two regions $X, Y$ are spacelike separated if every pair of points $(x, y) \in X \times Y$ is spacelike separated. The spacelike complement of a region $X$ is denoted by $X^{\prime}$.

Standard subspaces. Let $\mathcal{H}$ be a Hilbert space. A standard subspace $H$ of $\mathcal{H}$ is a closed, real linear subspace that is cyclic $(H+i H$ is dense) and separating $(H \cap i H=\{0\})$. Standard subspaces of the one-particle space naturally appear in the above free field construction: the standardness of $H_{\Phi}(O)$ is equivalent to the Reeh-Schlieder property that the vacuum vector is cyclic and separating for the corresponding local von Neumann algebras $\mathcal{A}(O)$ [1].

If $H$ is a standard subspace, the Tomita operator $S: \xi+i \eta \mapsto \xi-i \eta, \xi, \eta \in H$, remembers $H$ as $H=\operatorname{Ker}(S-1)$, and its polar decomposition $S=J \Delta^{1 / 2}$ gives the modular operator $\Delta$ and the modular conjugation $J$ that satisfy the one-particle version of the Tomita-Takesaki theorem:

$$
\Delta^{i t} H=H \quad \forall t \in \mathbb{R}, \quad J H=H^{\prime},
$$

where $H^{\prime}$ is the symplectic complement of $H$.

The Bisognano-Wichmann property. Now, let $U$ be a positive energy representation of $\mathcal{P}_{+}^{\uparrow}$ on $\mathcal{H}$, and $\mathcal{W} \ni W \longmapsto H(W)$ a net of standard subspaces on the wedge regions of the Minkowski spacetime $\mathbb{R}^{4}$, which is $U$-covariant:

$$
U(g) H(W)=H(g W) .
$$

The Bisognano-Wichmann property [2] asserts that the modular group of $H(W)$ is related to the boost transformations $\Lambda_{W}$ preserving $W$ :

$$
\Delta_{W}^{i t}=U\left(\Lambda_{W}(-2 \pi t)\right) .
$$

If $H(C)$ is cyclic for all cones $C$, then $J_{W}$ acts geometrically as a reflection around the edge of the wedge, so there exists an anti-unitary PCT operator

$$
\Theta \equiv U\left(R_{W}\right) J_{W}
$$

where $R_{W}$ is the spatial $\pi$-rotation mapping $W$ onto $W$. 
In quantum field theory, the Bisognano-Wichmann property pertains to the standard subspaces $\overline{\mathcal{A}(W)_{\text {s.a. }} \Omega}$, where $\mathcal{A}(W)_{\text {s.a. }}$ is the selfadjoint part of the von Neumann algebra of local observables in a wedge. It was established model-independently for large classes of quantum field theories, cf. Sect. 10.3. Because the modular group is characterized by the KMS property, its physical meaning is that the vacuum state is a KMS state for the boost subgroup, when restricted to the algebra of a Rindler wedge; in other words, the restriction of the vacuum state is a thermal state for the geodesic observer on the Rindler spacetime. By this feature it is closely related to the Hawking-Unruh effect. We therefore believe the Bisognano-Wichmann property to be of a most fundamental character, and refer to the final comment 10.3 and to [14] for a discussion of this important point.

Modular localization. The paper [4] provided a canonical construction of a local net $H_{U}$ of standard subspaces on the wedge regions of the Minkowski spacetime $\mathbb{R}^{4}$ associated with any unitary, positive energy, representation $U$ of the Poincare group (with antiunitary PCT operator $\Theta$ ). One defines $\Delta_{W}$ and $J_{W}$ by the Eqs. (2), (3), then sets $S_{W} \equiv$ $J_{W} \Delta_{W}^{1 / 2}$ and

$$
H_{U}(W) \equiv\left\{\xi \in \mathcal{H}: S_{W} \xi=\xi\right\} .
$$

Isotony of the assignment $W \longmapsto H_{U}(W)$ (i.e., $H_{U}\left(W_{1}\right) \subset H_{U}\left(W_{2}\right)$ whenever $W_{1} \subset$ $W_{2}$ ) follows from positivity of the energy. Moreover $H_{U}$ is local (or twisted-local if we consider representations of the cover of the Poincaré group), indeed $H_{U}$ is wedge dual: $H_{U}\left(W^{\prime}\right)=H_{U}(W)^{\prime}$.

This construction is intrinsic, depending only on the representation $U$ without reference to a quantum field. By construction, $H_{U}$ satisfies the Bisognano-Wichmann property.

Notice that any net $W \longmapsto H(W)$ on wedges defines closed, real linear subspaces associated with any region $X$ that is contained in some wedge:

$$
H(X) \equiv \bigcap_{\mathcal{W} \ni W \supset X} H(W)
$$

Obviously, these definitions respect isotony $\left(H\left(X_{1}\right) \subset H\left(X_{2}\right)\right.$ whenever $\left.X_{1} \subset X_{2}\right)$, and locality.

If $H(O)$ is cyclic for double cones $O$, then $H(W)$ defined by additivity from the double cones coincides with the original $H(W)$ (assuming the Bisognano-Wichmann property).

A general result [4] shows furthermore that $H_{U}(C)$ defined as in (5) from the canonical net (4) is standard for spacelike cones $C \in \mathcal{C}$, for every representation $U$.

If $U$ is a representation with finite spin/helicity, then the modular localization subspace $H_{U}(X)$ as in (5) agrees with the standard subspace $H_{\Phi}(O)$ defined by the free field one-particle construction recalled above, therefore $H_{U}(O)$ is standard for any double cone $O$, and in this case we explicitly see how the space $H(X)$ of particle states localized in a bounded region $X$ is cyclic.

We should also comment that the paper [4] deals with the bosonic case (true representation of $\mathcal{P}_{+}^{\uparrow}$ ); however, the fermionic case can be treated analogously with usual modifications (and quantization on the anti-symmetric Fock space).

Infinite spin particles cannot be localized in bounded regions. As recalled, in Wigner's classification of unitary, positive energy, irreducible representations of the Poincaré 
group [34], massless representations fit into two classes, the ones with finite spin (helicity) and the ones with infinite spin, according to the representations of the "little group", the double cover $\widetilde{E}(2)$ of the Euclidean group of the plane $E(2)$.

Let $U$ be a massless representation with infinite spin; the space $H_{U}(C)$ was shown to be standard (cyclic) for spacelike cones but it remained open whether there are non-zero vectors localized in bounded regions [12]. Generalized (stringlike) Wightman fields associated with $U$ were later constructed [27], but the above localization problem remained unsettled.

We shall show here that $H_{U}(X)$ is trivial if $X$ is bounded, say $X=O$ a double cone, namely

$$
H_{U}(O) \equiv \bigcap_{\mathcal{W} \ni \supset \supset O} H_{U}(W)=\{0\} .
$$

Quantum Field Theory, I. An immediate consequence is that the free field net $\mathcal{A}$ of local von Neumann algebras associated with a representation $U$ of $\mathcal{P}_{+}^{\uparrow}$ with infinite spin is well defined, the vacuum vector is cyclic for $\mathcal{A}(C)$ if $C$ is a spacelike cone, but $\mathcal{A}(O)=\mathbb{C} \cdot \mathbf{1}$ if $O$ is a double cone: there is no non-trivial observable localized in a bounded spacetime region.

It also follows that there are no compactly localized observables on the same Hilbert space that are relatively local w.r.t. the infinite spin free field net. The absence of such observables was recently also demonstrated within an explicit field theoretic ansatz [18].

An important more general corollary is that, if $\mathcal{B}$ is any (Fermi-)local net of von Neumann algebras on a Hilbert space, covariant under a unitary positive energy representation $U$ of the Poincaré group, with the vacuum vector being cyclic (Reeh-Schlieder property) for double cone algebras, then no infinite spin representation can appear in the irreducible direct integral decomposition of $U$ (up to measure zero), provided that $\mathcal{B}$ satisfies the fundamental Bisognano-Wichmann property [2].

This shows why infinite spin particles do not appear in a theory of local observables. They are however compatible with stringlike localization. At this point it is worth mentioning that localization in spacelike cones is natural in quantum field theory, indeed massive charges may always be localized in spacelike cones [5]. Low-dimensional nontrivial models with trivial local algebras are exhibited in [20].

Strategy of proof. Let $U$ be a unitary, massless irreducible representation of $\mathcal{P}_{+}^{\uparrow}$. The starting point is the observation that $U$ is dilation covariant if and only if it has finite spin. Assuming $H_{U}(O)$ to be standard for double cones $O$, we infer by the Huygens principle that $H_{U}\left(V_{+}\right)$is standard, where $V_{+}$is the forward light cone. By standard subspace analysis, in particular by using an analogue of Borchers' theorem [3,24], $\Delta_{H_{U}\left(V_{+}\right)}$has dilation commutation relations with $U$. So $U$ must have finite spin.

Extensions of results. Our results hold in any space dimension $s \geq 2$. As is known, if $s$ is even the Huygens principle doesn't hold and we need to work with a corresponding property of the wave equation that we haven't found in the literature. The case $s=2$ is peculiar as the infinite spin representations are not "infinite", namely they are associated with one-dimensional representations of the little group. The Fermi case, namely representations of a cover of $\mathcal{P}_{+}^{\uparrow}$, is also studied. We treat the case $s=3$ (the physical Minkowski spacetime) in detail and add a further section with the necessary analysis in different spacetime dimensions.

Quantum Field Theory, II. For interacting theories satisfying the Bisognano-Wichmann property, we show that the subspace $\overline{\mathcal{B}(O) \Omega}$ (independent of the double cone $O$ ) cannot 
carry an infinite spin representation. Thus, if the theory possesses infinite spin particles, then the vacuum vector cannot be cyclic for $\mathcal{B}(O)$, where $\mathcal{B}$ is the local net of von Neumann algebras describing our theory, i.e., the infinite spin particle states cannot be generated from the vacuum by operations in bounded spacetime regions.

Indeed, no infinite spin particle state can be obtained by adding to $\mathcal{B}$ a finite charge localized in a bounded spacetime region. In other words, no infinite spin representation can appear in the irreducible disintegration of the covariance unitary representation of DHR sectors of $\mathcal{B}$ (with finite statistics).

We emphasize that the Bisognano-Wichmann property is essential in the argument, by providing a counter-example without this property, in which free infinite spin particles exist with cyclic double cone algebras.

Thus, at least one (artificial) way to accomodate new physics involving observable infinite spin particles would consist in relaxing the Bisognano-Wichmann property-in spite of its very fundamental nature. More interesting is the picture (Sect. 10.1) that we obtain when we start with a (compactly) local observable net; we have a field algebra net that generates a non-trivial but non-cyclic subspace, an interacting theory with infinite spin particles; this structure exactly complies with the picture envisaged in [30].

\section{Standard Subspaces}

We begin by recalling some definitions and results on standard subspaces and their modular structures. Further details can be found in [24,25].

A linear, real, closed subspace $H$ of a complex Hilbert space $\mathcal{H}$ is called cyclic if $H+i H$ is dense in $\mathcal{H}$, separating if $H \cap i H=\{0\}$ and standard if it is cyclic and separating.

Given a standard subspace $H$ one defines the Tomita operator $S_{H}$, the closed, antilinear involution with domain $H+i H$, given by $S_{H}: \xi+i \eta \mapsto \xi+i \eta, \xi, \eta \in H$. The polar decomposition $S_{H}=J_{H} \Delta_{H}^{1 / 2}$ defines the positive selfadjoint modular operator $\Delta_{H}$ and the anti-unitary modular conjugation $J_{H} . \Delta_{H}$ is invertible and $J_{H} \Delta_{H} J_{H}=\Delta_{H}^{-1}$.

Pairs $(J, \Delta)$, where $J$ is an anti-unitary involution and $\Delta$ a selfadjoint positive invertible operator s.t. $J \Delta J=\Delta^{-1}$ are in 1-1 correspondence with closed, anti-linear, densely defined involutions $S=J \Delta^{1 / 2}$ and in 1-1 correspondence with standard subspaces $H=\operatorname{Ker}(S-1)$. by

If $H$ is a closed, real linear subspace of $\mathcal{H}$, the symplectic complement of $H$ is defined

$$
H^{\prime} \equiv\{\xi \in \mathcal{H} ; \Im(\xi, \eta)=0 \forall \eta \in H\}=(i H)^{\perp_{\mathbb{R}}},
$$

where $\perp_{\mathbb{R}}$ denotes the orthogonal in $\mathcal{H}$ viewed as a real Hilbert space with respect to the real part of the scalar product. $H^{\prime}$ is a closed, real linear subspace of $\mathcal{H}$ and $H=H^{\prime \prime}$.

$H$ is cyclic (separating) iff $H^{\prime}$ is separating (cyclic), thus $H$ is standard iff $H^{\prime}$ is standard and we have

$$
S_{H^{\prime}}=S_{H}^{*}
$$

The fundamental properties of the modular operator and conjugation are

$$
\Delta_{H}^{i t} H=H, \quad J_{H} H=H^{\prime}, \quad t \in \mathbb{R},
$$

and $t \mapsto \Delta_{H}^{i t}$ is called the one-parameter unitary modular group of $H$ (cf. [29,33]). 
Let $H$ be a real linear subspace of $\mathcal{H}$ and $V$ a one-parameter group of unitaries on $\mathcal{H}$ such that $V(t) H=H, t \in \mathbb{R}$. $V$ satisfies the $K M S$ condition with inverse temperature $\beta>0$ on $H$ if, for every given $\xi, \eta \in H$, there exists a function $F$, analytic on the strip $\{z \in \mathbb{C}: 0<\Im z<1\}$, bounded and continuous on its closure, such that:

$$
\begin{gathered}
F(t)=\langle\eta, V(t) \xi\rangle, \quad t \in \mathbb{R}, \\
F(t+i \beta)=\langle V(t) \xi, \eta\rangle, \quad t \in \mathbb{R} .
\end{gathered}
$$

Since the uniform limit of holomorphic functions is holomorphic, it follows that if the KMS condition holds on $H$, then it holds on the closure $\bar{H}$ of $H$.

Lemma 2.1 [24,25]. If $H \subset \mathcal{H}$ is a standard subspace, then $t \mapsto \Delta_{H}^{-i t}$ satisfies the KMS condition at inverse temperature 1.

Conversely, if $H$ is a closed, real linear, cyclic subspace of $\mathcal{H}$ and $V$ a one-parameter unitary group on $\mathcal{H}$ with $V(t) H=H, t \in \mathbb{R}$, satisfying the KMS condition on $H$ at inverse temperature 1 , then $H$ is standard and $V(t)=\Delta_{H}^{-i t}$.

The following lemma is a consequence of the KMS condition for the modular group.

Lemma 2.2 [24,25]. Let $H \subset \mathcal{H}$ be a standard subspace, and $K \subset H$ a closed, real linear subspace of $K$.

If $\Delta_{H}^{i t} K=K, \forall t \in \mathbb{R}$, then $K$ is a standard subspace of $\mathcal{K} \equiv \overline{K+i K}$ and $\left.\Delta_{H}\right|_{K}$ is the modular operator of $K$ on $\mathcal{K}$. If moreover $K$ is a cyclic subspace of $\mathcal{H}$, then $H=K$.

We shall also need the following basic lemma.

Lemma $2.3[24,25]$. Let $H \subset \mathcal{H}$ be a standard subspace, and $U$ a unitary on $\mathcal{H}$ such that $U H=H$. Then $U$ commutes with $\Delta_{H}$ and $J_{H}$.

The following is the one-particle analogue of Borchers' theorem [3].

Theorem 2.4 [24,25]. Let $H \subset \mathcal{H}$ be a standard subspace, and $U$ a one-parameter unitary group on $\mathcal{H}$ with positive generator, such that $U(t) H \subset H, t \geq 0$. Then $\Delta_{H}^{i s} U(t) \Delta_{H}^{-i s}=U\left(e^{-2 \pi s} t\right)$.

We now want to study the tensor product of standard subspaces. Let $H$ and $K$ be standard subspaces of the Hilbert spaces $\mathcal{H}$ and $\mathcal{K}$ respectively, and $S_{H}, S_{K}$ the associated Tomita operators. Then $S \equiv S_{H} \otimes S_{K}$ is a closed, densely defined anti-linear involution. Therefore $S=S_{M}$ where $M \equiv\{\xi \in \operatorname{Dom}(S): S \xi=\xi\}$ is a standard subspace of $\mathcal{H} \otimes \mathcal{K}$.

We define the tensor product of $H$ and $K$ by $H \otimes K \equiv M$; in other words $H \otimes K$ is defined through the formula

$$
S_{H \otimes K} \equiv S_{H} \otimes S_{K}
$$

Proposition 2.5. If $H$ and $K$ are standard subspaces of $\mathcal{H}$ and $\mathcal{K}$ respectively, we have

$$
(H \otimes K)^{\prime}=H^{\prime} \otimes K^{\prime} .
$$

Proof. Immediate from the equality

$$
S_{(H \otimes K)^{\prime}}=S_{H \otimes K}^{*}=\left(S_{H} \otimes S_{K}\right)^{*}=S_{H}^{*} \otimes S_{K}^{*}=S_{H^{\prime} \otimes K^{\prime}} .
$$


With $H, K$ real linear subspaces of $\mathcal{H}$ and $\mathcal{K}$ respectively we denote by $H \odot K$ the real linear span of $\{\xi \otimes \eta: \xi \in H, \eta \in K\}$.

Proposition 2.6. Let $H$ and $K$ be standard subspaces of $\mathcal{H}$ and $\mathcal{K}$. We have:

$$
H \otimes K=\overline{H \odot K}
$$

Proof. $\overline{H \odot K}$ is cyclic since $H \odot K+i H \odot K=(H+i H) \odot(K+i K)$, which is dense in $\mathcal{H} \otimes \mathcal{K}$. Clearly $H \odot K$ is in the domain of $S_{H} \otimes S_{K}=S_{H \otimes K}$, thus $H \odot K \subset H \otimes K$. Now $\Delta_{H \otimes K}^{i t}=\Delta_{H}^{i t} \otimes \Delta_{K}^{i t}$ leaves globally invariant $H \odot K$, hence $\overline{H \odot K}$. By Lemma 2.2 we conclude that $\overline{H \odot K}$ is equal to $H \otimes K$.

By Proposition 2.6, we may equivalently define the tensor product of the closed, real linear subspaces $H$ and $K$ of $\mathcal{H}$ and $\mathcal{K}$ by $H \otimes K \equiv \overline{H \odot K}$.

Given a family of real linear subspaces $H_{a}$ of $\mathcal{H}$, we shall denote by $\sum_{a} H_{a}$ the real linear span of the $H_{a}$ 's.

Lemma 2.7. Let $\left\{H_{a}\right\}$ be a family of closed, real linear subspaces of $\mathcal{H}$. Then

$$
\left(\bigcap_{a} H_{a}\right)^{\prime}=\overline{\sum_{a} H_{a}^{\prime}}
$$

Proof. We have

$$
\left(\bigcap_{a} H_{a}\right)^{\prime}=\left(i \bigcap_{a} H_{a}\right)^{\perp_{\mathbb{R}}}=\left(\bigcap_{a} i H_{a}\right)^{\perp_{\mathbb{R}}}=\overline{\sum_{a}\left(i H_{a}\right)^{\perp \mathbb{R}}}=\overline{\sum_{a} H_{a}^{\prime}}
$$

Lemma 2.8. Let $\left\{H_{a}\right\}$ and $\left\{K_{b}\right\}$ be families of standard subspaces of $\mathcal{H}$ and $\mathcal{K}$ respectively, and suppose both the intersections $H \equiv \bigcap_{a} H_{a}$ and $K \equiv \bigcap_{b} K_{b}$ to be cyclic. We have:

$$
H \otimes K=\bigcap_{a, b}\left(H_{a} \otimes K_{b}\right)
$$

Proof. By Lemma 2.7 we have to show that

$$
(H \otimes K)^{\prime}=\overline{\sum_{a, b}\left(H_{a} \otimes K_{b}\right)^{\prime}} .
$$

By Proposition 2.5, we have indeed:

$$
(H \otimes K)^{\prime}=H^{\prime} \otimes K^{\prime}=\overline{\sum_{a} H_{a}^{\prime}} \otimes \overline{\sum_{b} K_{b}^{\prime}}=\overline{\sum_{a, b} H_{a}^{\prime} \otimes K_{b}^{\prime}}=\overline{\sum_{a, b}\left(H_{a} \otimes K_{b}\right)^{\prime}} .
$$




\section{Massless Representations of the Poincaré Group}

For the benefit of the reader, we first deal within the case of the four-dimensional spacetime, later extending our results to different dimensions.

If $G$ is a locally compact group, $H \subset G$ a closed subgroup, and $V$ a unitary representation of $H$, we denote by $\operatorname{Ind}_{H \uparrow G} V$ the unitary representation of $G$ induced by $V$.

The Poincaré group $\mathcal{P}_{+}^{\uparrow}$ is the semi-direct product $\mathbb{R}^{4} \rtimes \mathcal{L}_{+}^{\uparrow}$ of the proper orthochronous Lorentz group $\mathcal{L}_{+}^{\uparrow}$ and the translation group $\mathbb{R}^{4}$, where $\mathcal{L}_{+}^{\uparrow}$ acts naturally on $\mathbb{R}^{4}$.

The universal cover $\widetilde{\mathcal{L}}_{+}^{\uparrow}$ of $\mathcal{L}_{+}^{\uparrow}$ is a double cover, isomorphic to $\operatorname{SL}(2, \mathbb{C})$. Accordingly, the universal cover $\widetilde{\mathcal{P}}_{+}^{\uparrow}$ of $\mathcal{P}_{+}^{\uparrow}$ is isomorphic to $\mathbb{R}^{4} \rtimes \operatorname{SL}(2, \mathbb{C})$.

One can choose the covering map $\sigma: \operatorname{SL}(2, \mathbb{C}) \rightarrow \mathcal{L}_{+}^{\uparrow}$, so that $\sigma$ maps the oneparameter subgroup $\alpha$

$$
\alpha(t)=\left(\begin{array}{cc}
e^{t / 2} & 0 \\
0 & e^{-t / 2}
\end{array}\right), \quad t \in \mathbb{R},
$$

to the one-parameter group of boosts in the $x_{3}$-direction, and $\sigma$ restricts to the usual covering map $\mathrm{SU}(2) \rightarrow \mathrm{SO}(3)$. Explicitly, one identifies a vector $x=\left(x_{0}, x_{1}, x_{2}, x_{3}\right) \in$ $\mathbb{R}^{4}$ with the matrix $X_{x}=\left(\begin{array}{cc}x_{0}+x_{3} & x_{1}-i x_{2} \\ x_{1}+i x_{2} & x_{0}-x_{3}\end{array}\right)$ and defines the Lorentz transformation $\sigma(A) \in$ $\mathcal{L}_{+}^{\uparrow}$ acting on $x$ through $X_{\sigma(A) x}=A X_{x} A^{*}, A \in \operatorname{SL}(2, \mathbb{C})$, see [32].

The translation group $\mathbb{R}^{4}$ is thus also a normal subgroup of $\widetilde{\mathcal{P}}_{+}^{\uparrow}$. According to the Mackey machine (see [37]), if $U$ is an irreducible unitary representation of $\widetilde{\mathcal{P}}_{+}^{\uparrow}$, then $U$ is induced by an irreducible unitary representation $U_{0}$ of $\overline{\operatorname{Stab}}_{p}$ :

$$
U=\operatorname{Ind}_{\overline{\operatorname{Stab}}_{p} \uparrow \widetilde{\mathcal{P}}_{+}^{\uparrow}} U_{0}
$$

here the momentum $p \in \mathbb{R}^{4}$ is a point in the dual group of the translations (i.e., a character), $\overline{\operatorname{Stab}}_{p}$ is the stabilizer of $p$ for the action of $\widetilde{\mathcal{P}}_{+}^{\uparrow}$ on the characters given by the adjoint action on their arguments, and $\left.U_{0}\right|_{\mathbb{R}^{4}}$ is the one-dimensional representation $p$.

Notice that $\widetilde{\mathcal{L}}_{+}^{\uparrow}$ acts naturally on $\mathbb{R}^{4}$ and $\mathbb{R}^{4}$ acts trivially on itself, so one has

$$
\overline{\operatorname{Stab}}_{p}=\mathbb{R}^{4} \rtimes \operatorname{Stab}_{p},
$$

where $\operatorname{Stab}_{p} \subset \widetilde{\mathcal{L}}_{+}^{\uparrow}$ is the stabiliser of $p$ in $\widetilde{\mathcal{L}}_{+}^{\uparrow}$ acting naturally on $\mathbb{R}^{4}$ (the little group). Points $p$ in the same $\mathcal{L}_{+}^{\uparrow}$-orbit give rise to equivalent representations.

We are interested in a positive energy, massless representation $U$, thus $p \in \partial V_{+}$the boundary of the forward light cone. We assume $U$ is not the identity, thus $p \neq 0$ and we shall choose and fix $p=q$ with

$$
q \equiv(1,0,0,1) \in \partial V_{+}
$$

$\left(\partial V_{+} \backslash\{0\}\right.$ is a $\mathcal{L}_{+}^{\uparrow}$-orbit).

Then $\operatorname{Stab}_{q}$, the little group of $(1,0,0,1)$, is isomorphic to $\widetilde{E}(2)$, the double cover of the Euclidean group of the plane $E(2)$ :

$$
\operatorname{Stab}_{q}=\left\{\left(\begin{array}{ll}
u & z \\
0 & \bar{u}
\end{array}\right): u, z \in \mathbb{C},|u|=1\right\} .
$$


The irreducible representation $U_{0}$ of $\overline{\mathrm{Stab}}_{p}$ in (7) has the form

$$
U_{0}(g, x)=V(g) q(x), \quad g \in \operatorname{Stab}_{q}, \quad x \in \mathbb{R}^{4},
$$

where $V$ is an irreducible representation of $\operatorname{Stab}_{q}=\widetilde{E}(2)$ and $q$ is the character of $\mathbb{R}^{4}$.

Now $\widetilde{E}(2)$ is the semi-direct product $\mathbb{R}^{2} \rtimes \mathbb{T}$ and an irreducible representation $V$ of $\widetilde{E}(2)$ fits in one of the following two classes:

(a) The restriction of $V$ to $\mathbb{R}^{2}$ is trivial;

(b) The restriction of $V$ to $\mathbb{R}^{2}$ is non-trivial.

Irreducible representations of $\widetilde{E}(2)$ in class (a) are thus labelled by the integers, the dual of $\mathbb{T}$, while irreducible representations in class (b) are labelled by $\kappa>0$, the radius of a circle in $\mathbb{R}^{2}$, the joint spectrum of the $\widetilde{E}(2)$-translations.

We say in case (a) that $U$ has finite spin (or finite helicity); in case (b) that $U$ has infinite spin. Therefore an irreducible, infinite spin representation $U$ of $\widetilde{\mathcal{P}}_{+}^{\uparrow}$ has the form

$$
U_{\kappa, \varepsilon}=\operatorname{Ind}_{\operatorname{Stab}_{q} \uparrow \widetilde{\mathcal{P}}_{+}^{\uparrow}} \bar{V}_{\kappa, \varepsilon}
$$

where $\bar{V}_{\kappa, \varepsilon}$ is given by (9):

$$
\bar{V}_{\kappa, \varepsilon}(g, x)=V_{\kappa, \varepsilon}(g) q(x), \quad g \in \widetilde{E}(2), \quad x \in \mathbb{R}^{4},
$$

with $V=V_{\kappa, \varepsilon}$ is the representation of $\widetilde{E}(2)$ in which the spectrum of the translations is the circle of radius $\kappa>0$, and the rotation by $2 \pi$ is represented by $+\mathbf{1}$ (bosonic case, $\varepsilon=0$ ) resp. by $-\mathbf{1}$ (fermionic case, $\varepsilon=\frac{1}{2}$ ); so infinite spin representations are labelled by $\kappa>0$ and $\varepsilon=0, \frac{1}{2}$. We shall denote by $\tau(z), z \in \mathbb{C}$, the element of $\widetilde{E}(2) \subset \operatorname{SL}(2, \mathbb{C})$ given by

$$
\tau(z)=\left(\begin{array}{ll}
1 & z \\
0 & 1
\end{array}\right)
$$

the two translation one-parameter subgroups of $\widetilde{E}(2)$ are $\mathbb{R} \ni x \mapsto \tau(x)$, and $\mathbb{R} \ni y \mapsto$ $\tau($ iy) and we have the commutation relations

$$
\alpha(t) \tau(z) \alpha(t)^{-1}=\tau\left(e^{t} z\right) .
$$

\section{Infinite Spin Representations are Not Dilation Covariant}

As is known, an irreducible, massless finite helicity unitary representation extends, on the same Hilbert space, to a representation of the group of transformations of the Minkowski spacetime generated by $\widetilde{\mathcal{P}}_{+}^{\uparrow}$ and dilations (indeed to a unitary representation of the conformal group). We show here that irreducible infinite spin representations are not dilation covariant in this sense. We suppress the Bose/Fermi label $\varepsilon$ which is irrelevant for the issue at hand.

Lemma 4.1. Let $G$ be a locally compact group, $H \subset G$ a closed subgroup and $\beta$ an automorphism of $G$ such that $\beta(H)=H$. If $V$ is a unitary representation of $H$ and $U \equiv \operatorname{Ind}_{H \uparrow G} V$, then

$$
U \cdot \beta=\operatorname{Ind}_{H \uparrow G} V \cdot \beta_{0}
$$

where $\left.\beta_{0} \equiv \beta\right|_{H}$. 
Proof. The lemma follows by the unicity of the induced representation, a consequence of the unicity of the measure class of a quasi-invariant Borel measure on $H \backslash G$.

Corollary 4.2. Let $U_{\kappa}=\operatorname{Ind}_{\mathbb{R}^{4} \rtimes \widetilde{E}(2) \uparrow \widetilde{\mathcal{P}}_{+}^{\uparrow}} \bar{V}_{\kappa}$ be an infinite spin, irreducible unitary representation of $\widetilde{\mathcal{P}}_{+}^{\uparrow}$, and $\beta$ an automorphism of $\widetilde{\mathcal{P}}_{+}^{\uparrow}$ preserving the element $q$ of (the dual of) the translation subgroup. Then $\beta\left(\operatorname{Stab}_{q}\right)=\operatorname{Stab}_{q}$ and

$$
U_{\kappa} \cdot \beta=U_{\kappa_{\beta}}\left(\equiv \operatorname{Ind}_{\mathbb{R}^{4} \rtimes \widetilde{E}(2) \uparrow \widetilde{\mathcal{P}}_{+}^{\uparrow}} \bar{V}_{\kappa_{\beta}}\right),
$$

where $\kappa_{\beta}$ is given by $V_{\kappa_{\beta}}=V_{\kappa} \cdot \beta_{0}$ with $\beta_{0}$ the automorphism of $\widetilde{E}(2)$ given by $\beta_{0}=$ $\left.\beta\right|_{\operatorname{Stab}_{q}}$

Proof. This follows from Lemma 4.1.

We shall say that a unitary representation $U$ of $\widetilde{\mathcal{P}}_{+}^{\uparrow}$ on the Hilbert space $\mathcal{H}$ is dilation covariant if $U$ extends to a unitary representation on $\mathcal{H}$ of the group generated by $\widetilde{\mathcal{P}}_{+}^{\uparrow}$ and dilations. Namely there exists a one-parameter unitary group $D(t)$ on $\mathcal{H}$ such that $D$ commutes with $\left.U\right|_{\widetilde{\mathcal{L}}_{+}}$and

$$
D(t) U(x) D(-t)=U\left(e^{t} x\right),
$$

for $x$ in the translation group $\mathbb{R}^{4}$.

Proposition 4.3. Let $U$ be an irreducible, positive energy, unitary representation of $\widetilde{\mathcal{P}}_{+}^{\uparrow}$. Then $U$ is dilation covariant iff $U$ is massless with finite spin.

Proof. Let $\delta_{t}$ the the automorphism of $\widetilde{\mathcal{P}}_{+}^{\uparrow}$ given by $\delta_{t}(g)=g$ if $g \in \widetilde{\mathcal{L}}_{+}$and $\delta_{t}(p)=e^{t} p$ if $p \in \mathbb{R}^{4}$. We want to show that $U$ is inequivalent to $U \cdot \delta_{t}, t \neq 0$, if $U$ is irreducible with infinite spin.

Let then $U=U_{\kappa}$ be given by (7), namely $U_{\kappa}=\operatorname{Ind}_{\mathbb{R}^{4} \rtimes \widetilde{E}(2) \uparrow \widetilde{\mathcal{P}}_{+}^{\uparrow}} \widetilde{V}_{\kappa}$. We shall show that

$$
U_{\kappa} \cdot \delta_{t}=U_{e^{-t} \kappa} .
$$

This will prove the Proposition because $U_{\kappa}$ and $U_{\kappa^{\prime}}$ are inequivalent if $\kappa \neq \kappa^{\prime}$.

Now let $\alpha_{t}$ be the lift to $\widetilde{\mathcal{P}}_{+}^{\uparrow}$ of the inner one-parameter automorphism group of $\mathcal{P}_{+}^{\uparrow}$ implemented by the boost in 3-direction, namely $\alpha$ is given by Eq. (6). Then

$$
\alpha_{t}(q)=\delta_{t}(q)=\left(e^{t}, 0,0, e^{t}\right),
$$

where $q=(1,0,0,1)$ as above. Thus the automorphisms

$$
\beta_{t} \equiv \alpha_{-t} \cdot \delta_{t}
$$

fix $q$. Since $\alpha_{-t}$ is inner, we have $U_{\kappa} \cdot \alpha_{-t}=U_{\kappa}$, thus

$$
U_{\kappa} \cdot \delta_{t}=U_{\kappa} \cdot \alpha_{-t} \cdot \delta_{t}=U_{\kappa} \cdot \beta_{t} .
$$

We now apply Corollary 4.2 and see that

$$
U_{\kappa} \cdot \delta_{t}=U_{\kappa^{\prime}}
$$

where $\kappa^{\prime}$ is given by

$$
V_{\kappa^{\prime}}=\left.V_{\kappa} \cdot \beta_{t}\right|_{\widetilde{E}(2)}=\left.V_{\kappa} \cdot \alpha_{-t}\right|_{\widetilde{E}(2)},
$$

thus $\kappa^{\prime}=e^{-t} \kappa$ by the commutation relation (11) [36, Lemma 4]. 


\section{Double Cone Localization Implies Dilation Covariance}

Let $U$ be a unitary, positive energy representation of the cover $\widetilde{\mathcal{P}}_{+}^{\uparrow}$ of the Poincaré group on a Hilbert space $\mathcal{H}$.

A $U$-covariant net of standard subspaces $\mathcal{H}$ on the set $\mathcal{W}$ of wedge regions of the Minkowski spacetime is a map

$$
H: \mathcal{W} \ni W \longmapsto H(W) \subset \mathcal{H}
$$

that associates a closed real linear subspace $H(W)$ with each $W \in \mathcal{W}$, satisfying:

1. Isotony: if $W_{1} \subset W_{2}$ then $H\left(W_{1}\right) \subset H\left(W_{2}\right)$;

2. Poincaré covariance: $U(g) H(W)=H(g W), g \in \widetilde{\mathcal{P}}_{+}^{\uparrow}$;

3. Reeh-Schlieder property: $H(W)$ is cyclic $\forall W \in \mathcal{W}$;

4. Bisognano-Wichmann property:

$$
\Delta_{H(W)}^{i t}=U\left(\Lambda_{W}(-2 \pi t)\right), \quad \forall W \in \mathcal{W} ;
$$

5. Twisted locality: For every wedge $W \in \mathcal{W}$ we have

$$
Z H\left(W^{\prime}\right) \subset H(W)^{\prime}
$$

with $Z$ unitary, $Z=\frac{\mathbf{1}+i \Gamma}{1+i}$.

Due to twisted locality, each $H(W)$ is indeed a standard subspace, so the modular operators in Property 4 are defined.

Here $\Gamma \equiv U(2 \pi)$, the unitary corresponding to a $2 \pi$ spatial rotation in the representation $U$, namely $\Gamma$ is the image under $U$ of the non-trivial element in the centre of $\widetilde{\mathcal{L}}_{+}^{\uparrow}$. Clearly $\Gamma$, hence $Z$, commutes with $U$.

Notice that if $U$ is bosonic $(\Gamma=\mathbf{1})$, then $Z=\mathbf{1}$, and twisted locality is locality. If $U$ is fermionic $(\Gamma=-\mathbf{1})$, then $Z=-i$ and $H\left(W^{\prime}\right) \subset i H(W)$.

Lemma 2.2 then implies twisted duality for wedges:

$$
H\left(W^{\prime}\right)=Z H(W)^{\prime} .
$$

Starting with a $U$-covariant net $H$ on $\mathcal{W}$ as above, one gets a net of closed, real linear subspaces on double cones $O$ defined by

$$
H(O) \equiv \bigcap_{\mathcal{W} \ni W \supset O} H(W) .
$$

Note that $H(O)$ is not necessarily cyclic. If $H(O)$ is cyclic, then

$$
H(W)=\overline{\sum_{O \subset W} H(O)}
$$

by Lemma 2.2.

The following proposition is proved in [4], (ii) $\Rightarrow$ (i), and in [12], (i) $\Rightarrow$ (ii), for nets of von Neumann algebras; yet the same argument gives a proof in the standard subspace setting.

Proposition 5.1 [4,12]. Let $H$ be a $U$-covariant net of standard subspaces of $\mathcal{H}$ as above (properties 1-5). The following are equivalent: 
(i) $H(C) \equiv \bigcap_{\mathcal{W} \ni \supset C} H(W)$ is cyclic for all spacelike cones $C$;

(ii) $U$ extends to an (anti-)unitary representation $\hat{U}$ of $\widetilde{\mathcal{P}}_{+}$on $\mathcal{H}$ and $H$ is the canonical net $H_{\hat{U}}$ associated with $\hat{U}$ (Eq. (4)).

Thus (in even spacetime dimension), with the above cone cyclicity assumption, there is an anti-unitary PCT operator.

The following proposition ensures a variant of the Reeh-Schlieder property. If $O, \widetilde{O}$ are double cones, we write $O \Subset \widetilde{O}$ if the closure of $O$ is contained in the interior of $\widetilde{O}$.

Proposition 5.2. Let $H(O)$ be defined as above in (13), with $U$ irreducible. If $H(O) \neq$ $\{0\}$ for some double cone $O$, then $H(\widetilde{O})$ is cyclic for every double cone $O \Subset \widetilde{O}$.

Proof. Let $O \Subset \widetilde{O}$ be double cones with $H(O) \neq\{0\}$ and $\xi$ a vector orthogonal to $H(\widetilde{O})$. We can find a $\delta>0$ s.t. $x+O \subset \widetilde{O}$, so

$$
f(x) \equiv\langle\xi, U(x) Z \eta\rangle=0,
$$

for $|x|<\delta$ and $\eta \in H(O)$, where $U(x)$ is the unitary translation by $x$. By positivity of the energy, $f$ has an analytic continuation on the tube $\mathbb{R}^{4}-i V^{+}$. Since $f(x)=0$ on an open subset of the boundary, by the Edge of the Wedge theorem $f$ is identically zero. Thus $\xi$ is orthogonal to all translates $H(O+x)$.

We consider now a wedge $W \supset \widetilde{O}$ and the corresponding boost one-parameter group $\Lambda_{W}$. By the KMS property entailed by the Bisognano-Wichmann property, there exists an analytic extension of the function $h$ :

$$
h(s) \equiv\left\langle\xi, U\left(\Lambda_{W}(2 \pi s)\right) Z \eta\right\rangle,
$$

on the strip $\{z \in \mathbb{C}: 0<\Im z<1\}$. Because $O \Subset \widetilde{O}, h(s)$ is zero for small real values of $s$. Thus the whole extension of $h$ has to be zero. It follows that

$$
\xi \perp H(g O), \quad \forall g \in \mathcal{P}_{+}^{\uparrow} .
$$

Now the closed, complex linear span generated by $\left\{H(g O): g \in \mathcal{P}_{+}^{\uparrow}\right\}$ is a $U$-invariant, non-zero, closed linear subspace of $\mathcal{H}$, that must be equal to $\mathcal{H}$ since $U$ is irreducible. Thus $\xi=0$ and $H(\widetilde{O})$ is cyclic.

Lemma 5.3. Assume that $U$ is a massless, unitary representation of $\widetilde{\mathcal{P}}_{+}^{\uparrow}$ acting covariantly on a twisted-local net of closed, real linear subspaces on double cones. Let $\mathrm{O}_{1}, \mathrm{O}_{2}$ be double cones with $\mathrm{O}_{2}$ in the timelike complement of $\mathrm{O}_{1}$, then

$$
H\left(O_{2}\right) \subset Z H\left(O_{1}\right)^{\prime} \text {. }
$$

Proof. Let $O_{r}$ be the double cone of radius $r>0$ centred at the origin, namely $O_{r}$ is the causal envelope of the ball of radius $r$ centred at the origin in the time zero hyperplane. Consider the two point function

$$
f(x)=\langle\xi, U(x) Z \eta\rangle, \quad \xi, \eta \in H\left(O_{r}\right) .
$$

Then $\square f=0$, namely $f$ is a solution of the wave equation, since the Fourier transform of $f$ (w.r.t. the Minkowski metric) is a measure with support in $\partial V_{+}$. In particular $\square \mathfrak{s} f=0$. Now $\mathfrak{s} f(x)=0$ if $x \in O_{2 r}^{\prime}$, because $O_{r}+x \subset O_{r}^{\prime}$. Thus, by the Huygens principle for solutions of the wave equations, also $\Im f(x)=0$ if $x$ belongs to the timelike complement of $O_{2 r}$. Thus $H\left(O_{r}+x\right) \subset Z H\left(O_{r}\right)^{\prime}$ for such $x$, namely for $x$ such that $O_{r}+x$ is contained in the timelike complement of $O_{r}$. This entails the thesis as $r>0$ is arbitrary. 
Proposition 5.4. Let $U$ be a massless representation of $\widetilde{\mathcal{P}}_{+}^{\uparrow}$, acting covariantly on a net $H$ of standard subspaces on wedges satisfying properties 1-5. If $H(O)$ is cyclic for some double cone $O$, then $U$ is dilation covariant.

If $U$ is irreducible, the same conclusion holds by assuming that $H(O) \neq\{0\}$ for some double cone $O$.

Proof. Let $H\left(V_{+}\right)$be the closed, real linear subspace generated by $H(O)$ as $O$ runs in the double cones contained in $V_{+}$, and similarly for $H\left(V_{-}\right) . H\left(V_{+}\right)$(and $H\left(V_{-}\right)$) is cyclic as it contains a cyclic real linear subspace $H(O)$ by assumptions (if $H(O)$ is cyclic, all its translated are cyclic). Since $H\left(V_{+}\right) \subset Z H\left(V_{-}\right)^{\prime}$ by Proposition 5.4, $H\left(V_{+}\right)$and $H\left(V_{-}\right)$are also separating, hence standard subspaces. Set

$$
D(2 \pi t)=\Delta_{H\left(V_{+}\right)}^{-i t}, \quad t \in \mathbb{R} .
$$

Then, by Lemma 2.3, $D(t)$ commutes with $U(g)$ if $g$ is in the Lorentz group, because $g V_{+}=V_{+}$, so $U(g) H\left(V_{+}\right)=H\left(V_{+}\right)$.

Thanks to positivity of the energy, the one-particle version of Borchers' theorem (Theorem 2.4) applies to all one-parameter groups of timelike translations. Since the latter generate all translations, we conclude that $D(s)$ scales the translations:

$$
D(s) U(x) D(-s)=U\left(e^{s} x\right), \quad s \in \mathbb{R},
$$

if $x$ is in the translation group. Thus $U$ is dilation covariant, with dilation unitaries $D(t)$.

The statement for $U$ irreducible then follows immediately by Proposition 5.2.

\section{Infinite Spin States are Not Localized in Bounded Regions}

We give here our main result.

Theorem 6.1. Let $U$ be an irreducible unitary, positive energy, massless, infinite spin representation of $\widetilde{\mathcal{P}}_{+}^{\uparrow}$ on a Hilbert space $\mathcal{H}$, and $H: \mathcal{W} \ni W \longmapsto H(W) \subset \mathcal{H}$ a $U$-covariant net of standard subspaces satisfying properties $1-5$. Then

$$
H(O) \equiv \bigcap_{\mathcal{W} \ni W \supset O} H(W)=\{0\},
$$

for every double cone $O \in \mathcal{O}$.

Proof. If $H(O) \neq\{0\}$ for some double cone $O$, then by Proposition $5.4 U$ must be dilation covariant, which is not possible by Proposition 4.3.

The consequences of this theorem in Quantum Field Theory will be discussed in Sect. 9.

\section{A Counter-Example}

In this section, we are going to see how dilation covariance and the double cone ReehSchlieder property for infinite spin (reducible) representations may both hold if the Bisognano-Wichmann property fails. We shall indeed show that a multiple of the direct integral

$$
\int_{\mathbb{R}_{+}}^{\oplus} U_{\kappa} d \kappa
$$


over all irreducible representations $U_{\kappa}$ of $\mathcal{P}_{+}^{\uparrow}$ of infinite spin $\kappa$ is dilation covariant and admits a local covariant net of standard subspaces, cyclic on double cones. Similar examples were put forward in $[28,35]$.

For the sake of the example, it is sufficient to consider representations $V$ of $\operatorname{SL}(2, \mathbb{C})$ that factor through $\mathcal{L}_{+}^{\uparrow}$, i.e., $V(1)=V(-1)$. Namely, $V$ is a true representation of $\mathcal{L}_{+}^{\uparrow}$. Since the choice of the pre-image of the covering map $\sigma$ does not matter in true representations, we shall identify $A \in \operatorname{SL}(2, \mathbb{C})$ with $\sigma(A) \in \mathcal{L}_{+}^{\uparrow}$ in this section, and again suppress the corresponding label $\varepsilon=0$.

The subgroup $\widetilde{E}(2) \subset \operatorname{SL}(2, \mathbb{C})$, the pre-image of $E(2)$ through $\sigma$, is given by (8).

Let $U_{0}$ be the unitary, massless, zero helicity, representation of the Poincaré group and $V$ a real unitary representation $\mathcal{L}_{+}^{\uparrow}$ on the Hilbert spaces $\mathcal{H}$ and $\mathcal{K}$ respectively. With $J$ an anti-unitary involution on $\mathcal{K}$ commuting with $V$, the vectors fixed by $J$ form a standard subspace $K$ of $\mathcal{K}$ and $V\left(\mathcal{L}_{+}^{\uparrow}\right) K=K, J_{K}=J, \Delta_{K}=\mathbf{1}$. In particular the constant net of standard subspaces $K(W) \equiv K$ is $V$-covariant.

We consider $V$ as a representations of $\mathcal{P}_{+}^{\uparrow}$ where the translation group acting identically.

Consider the following net of standard subspaces of $\mathcal{K} \otimes \mathcal{H}$

$$
H_{I}: \mathcal{W} \ni W \longmapsto H_{I}(W) \equiv K \otimes H(W) \subset \mathcal{K} \otimes \mathcal{H}
$$

where $H \equiv H_{U_{0}}$ is the canonical net associated with $U_{0}$. There are two unitary representations of the $\mathcal{P}_{+}^{\uparrow}$ on $\mathcal{K} \otimes \mathcal{H}$ :

$$
U_{V} \equiv V \otimes U_{0}
$$

and

$$
U_{I} \equiv I \otimes U_{0}
$$

where $I$ is the identity representation of $\mathcal{P}_{+}^{\uparrow}$ on $\mathcal{K}$. Clearly $U_{V}$ and $U_{I}$ are massless representations, as the energy-momentum spectrum is that of $U_{0}$.

$H_{I}$ is the canonical net associated with $U_{I}$. The net $H_{I}$ is both $U_{V}$-covariant and $U_{I}$-covariant. Only $U_{I}$ satisfies the Bisognano-Wichmann property as, by Lemma 2.6, the modular operator of $K \otimes H(W)$ is $\mathbf{1} \otimes \Delta_{H(W)}$. Then by Lemma 2.8

$$
H_{I}(O)=\bigcap_{W \supset O} H_{I}(W)=K \otimes \bigcap_{W \supset O} H(W)
$$

is cyclic, since $\bigcap_{W \supset O} H(W)$ is cyclic in $\mathcal{H}$.

So we have shown the following.

Proposition 7.1. The net $H_{I}$ of standard subspaces is local, $U_{V}$-covariant, and cyclic on double cones. $U_{V}$ decomposes into a direct integral of infinite spin representation. $U_{V}$ does not satisfy the Bisognano-Wichmann property.

We notice that the canonical net $H_{V}$ associated with $U_{V}$ is not covariant under the representation $U_{I}$.

We will now show that $U_{V}$ decomposes in a direct integral of infinite spin representations if $V$ does not contain the trivial representation.

Let $V_{+} \backslash\{0\} \ni p \mapsto B_{p} \in \mathcal{L}_{+}^{\uparrow}$ be a continuous map, with $B_{p}$ a Lorentz transformation mapping $q=(1,0,0,1)$ to $p$. 
We can identify as usual the elements of $\mathcal{H}$ with $L^{2}$-functions on $\partial V_{+} \backslash\{0\}$ w.r.t. the Lorentz invariant measure, thus elements of $\mathcal{K} \otimes H$ with $\mathcal{K}_{0}$-valued $L^{2}$-functions.

The following unitary operator

$$
\mathcal{K} \otimes \mathcal{H} \ni(p \mapsto \phi(p)) \longmapsto\left(p \mapsto V\left(B_{p}^{-1}\right) \phi(p)\right) \in \mathcal{K} \otimes \mathcal{H}
$$

intertwines $U_{V}$ with the representation $U_{V}^{\prime}$ given by

$$
\left(U_{V}^{\prime}(a, A) \phi\right)(p)=e^{i a \cdot p} V\left(B_{p}^{-1} A B_{A^{-1} p}\right) \phi\left(A^{-1} p\right), \quad \phi \in \mathcal{H}
$$

Since $B_{p}^{-1} A B_{A^{-1} p} \in \operatorname{Stab}_{q}=E(2)$ we may consider the irreducible disintegration of $\left.V\right|_{E(2)}$, then $U_{V}^{\prime}$, thus $U_{V}$, will accordingly disintegrate.

Since $\operatorname{SL}(2, \mathbb{C})$ is a simple, connected, non-compact Lie group with finite centre, the vanishing of the matrix coefficients theorem by Howe-Moore [37] ensures that $\lim _{g \rightarrow \infty}\langle\xi, V(g) \eta\rangle=0$, for all $\xi, \eta \in \mathcal{K}$, if $V$ does not contain the identity representation.

Lemma 7.2. Let $V$ be a unitary representation of $\mathcal{L}_{+}^{\uparrow}$ not containing the identity representation. Then $\left.V\right|_{E(2)}$ is a multiple of $\int_{\mathbb{R}_{+}}^{\oplus} V_{\kappa} d \kappa$, where $V_{\kappa}$ is the unitary irreducible representation of $E(2)$ with radius $\kappa$.

Proof. By the vanishing of the matrix coefficients theorem, there is no non-zero vector fixed by $V \cdot \tau$, thus no radius zero representation appears in the irreducible direct integral decomposition of $\left.V\right|_{E(2)}$, namely $\left.V\right|_{E(2)}=\int_{\mathbb{R}} m(\kappa) V_{\kappa} d \mu(\kappa)$, where $m(\kappa)$ is the multiplicity function and $\mu$ is a Borel measure on $\mathbb{R}_{+}$.

The one-parameter subgroup $\alpha$ of $\operatorname{SL}(2, \mathbb{C})$ given in $(8)$ acts as dilation on the translations $\tau$, Eq. (11), thus

$$
\left.V\right|_{E(2)}=\int_{\mathbb{R}}^{\oplus} m(\kappa) V_{\kappa} d \mu(\kappa)=\int_{\mathbb{R}}^{\oplus} m(\kappa) V_{e^{t} \kappa} d \mu(\kappa)=\int_{\mathbb{R}}^{\oplus} m\left(e^{-t} \kappa\right) V_{\kappa} d \mu_{t}(\kappa)
$$

where $\mu_{t}(\kappa) \equiv \mu\left(e^{-t} \kappa\right)$, and this implies that $\mu_{t}$ is equivalent to $\mu$ (thus $\mu$ is equivalent to the Lebesgue measure) and $m$ constant $\mu$-almost everywhere.

The following Proposition is a consequence of the above Lemma.

Proposition 7.3. $U_{V}$ is a multiple of $\int_{\mathbb{R}_{+}}^{\oplus} U_{\kappa} d \kappa$, where $U_{\kappa}$ is the infinite spin, radius $\kappa$ representation of $\mathcal{P}_{+}^{\uparrow}$.

Proof. One considers the disintegration of $\left.V\right|_{E(2)}$ obtained in Lemma 7.2 and concludes the thesis by formula (15).

\section{Extensions to Spacetime Dimension $s \geq 2$}

In this section we are going to extend Propositions 4.3 and 5.4, and hence also Theorem 6.1 , in any spacetime dimensions $s \geq 2$. 
8.1. Dilation covariance. We begin by discussing the dilation covariance property.

The proper Lorentz group is $\mathcal{L}_{+} \equiv \mathcal{L}_{+}(s)=\mathrm{SO}(1, s)$, i.e., the group of $d \times d$ real matrices $A$ preserving the Minkowski metric $\langle 1,-1, \ldots,-1\rangle . \mathcal{L}_{+}$has two connected components and we denote by $\mathcal{L}_{+}^{\uparrow}$ the connected component of the identity.

$\mathcal{L}_{+}^{\uparrow}$ is not simply connected when $s>1$. Any element in $\mathcal{L}_{+}^{\uparrow}$ is the product of a rotation and a boost, so $\mathcal{L}_{+}^{\uparrow}$ is homotopy equivalent to $\mathrm{SO}(s)$, whose first homotopy group is $\mathbb{Z}_{2}$ if $s>2$ and $\mathbb{Z}$ if $s=2$ (see [19]). Therefore the universal covering $\widetilde{\mathcal{L}}_{+}^{\uparrow}$ of $\mathcal{L}_{+}^{\uparrow}$ is a double covering for $s>2$, whereas it is an infinite sheet covering if $s=2$. We shall thus treat the case $s=2$ separately.

The proper orthochronous Poincare group $\mathcal{P}_{+}^{\uparrow} \equiv \mathcal{P}_{+}^{\uparrow}(s)$ is the semi-direct product of $\mathcal{P}_{+}^{\uparrow} \equiv \mathbb{R}^{s+1} \rtimes \mathcal{L}_{+}^{\uparrow}$, with the natural action of $\mathcal{L}_{+}^{\uparrow}$ on $\mathbb{R}^{s+1}$.

We shall consider unitary representations of the universal covering group $\widetilde{\mathcal{P}}_{+}^{\uparrow}=$ $\mathbb{R}^{s+1} \rtimes \widetilde{\mathcal{L}}_{+}^{\uparrow}$, as they correspond to the projective unitary, positive energy representations of $\mathcal{P}_{+}^{\uparrow}$.

We are interested here in an irreducible, positive energy, massless representation $U$ of $\widetilde{\mathcal{P}}_{+}^{\uparrow}$. We choose and fix the point $q \equiv q_{s}=(1,0, \ldots, 0,1)$ in the Lorentz orbit $\partial V_{+} \backslash\{0\}$. If $U$ is non-trivial, then $U$ is associated with a unitary, irreducible representation of the little group of $q$, by inducing representations as in Sect. 3 .

The little group of $q$, namely the stabiliser subgroup of $\widetilde{\mathcal{L}}_{+}^{\uparrow}$ for the action of $\mathcal{L}_{+}^{\uparrow}$ on $\mathbb{R}^{s+1}$, is isomorphic to $\widetilde{E}(s-1)$, the double cover of the Euclidean group $E(s-1)$ on $\mathbb{R}^{s-1}, s>2$, i.e., $E(s-1)$ is the semi-direct product $\mathbb{R}^{s-1} \rtimes \mathrm{SO}(s-1)$. If $s=2$, the little group is the abelian group $\mathbb{R}$. We now assume $s>2$, afterwords we shall indicate the modifications in the $s=2$ case.

Every unitary representation $V$ of $\widetilde{E}(s-1)=\mathbb{R}^{s-1} \rtimes \widetilde{\mathrm{SO}}(s-1)$ is now induced by a unitary representation of the stabiliser of a point in $\mathbb{R}^{s-1}$ (for the adjoint action of $\widetilde{E}(s-1))$. Points in the same orbit give equivalent representations. The orbits in $\mathbb{R}^{s-1}$ under the natural $\mathrm{SO}(s-1)$ action are spheres of radius $\kappa \geq 0$. Such radii define inequivalent classes of unitary representations. As in the $3+1$-dimensional case, there are two cases:

- the restriction $\left.V\right|_{\mathbb{R}^{s-1}}$ is trivial $(\kappa=0)$;

- the restriction $\left.V\right|_{\mathbb{R}^{s-1}}$ is non-trivial $(\kappa>0)$.

If $U$ is associated, by induction, with a representation $V$ of the little group $\widetilde{E}(s-1)$ with $\kappa=0$ we say that $U$ has finite helicity, in the case $\kappa>0$ we say that $U$ has infinite spin.

With $V$ an irreducible representation of $\widetilde{E}(s-1)$ of radius $\kappa>0$ as above, $s>2$, the joint spectrum of the $\widetilde{E}(s-1)$-translation generators is the sphere in $\mathbb{R}^{s-1}$ of radius $\kappa$. Therefore

$$
\operatorname{spec}(i V(X))=[-\kappa, \kappa]
$$

where $X$ is any generator of the $E(s-1)$-translations and $V(X)$ the corresponding translation generator in the representation $V$.

We show now that infinite spin representations are not dilation covariant:

Proposition 8.1. Let $U$ be an irreducible, positive energy, unitary representation of $\widetilde{\mathcal{P}}_{+}^{\uparrow}(s), s \geq 2$. Then $U$ is dilation covariant iff $U$ is massless with finite spin.

Proof. We have seen in Proposition 4.3 in the case $s=3$ that

$$
\beta_{t}(X)=e^{-t} X
$$


where $\beta_{t}$, the automorphisms of $\mathcal{P}_{+}^{\uparrow}$ defined in (12), here acting on the Lie algebra of $\mathcal{P}_{+}^{\uparrow}$, and $X$ is a translation generator on the Lie algebra $\mathfrak{l i e}(E(2))$.

Now assume $s \geq 3$. The inclusion $\mathcal{P}_{+}^{\uparrow}(3) \subset \mathcal{P}_{+}^{\uparrow}(s)$ restricts to an inclusion $E(2) \subset$ $E(s-1)$ hence we have inclusions of Lie algebras $\mathfrak{l i e}(E(2)) \subset \mathfrak{l i e}(E(s-1)) \subset \mathfrak{l i e}\left(\mathcal{P}_{+}^{\uparrow}\right)$.

We consider the automorphisms $\beta_{t}$ of $\widetilde{\mathcal{P}}_{+}^{\uparrow}(s)$ analogously defined w.r.t. the zero and $s$ coordinates (the natural extension of $\beta_{t}$ from $\widetilde{\mathcal{P}}_{+}^{\uparrow}(3)$ to $\widetilde{\mathcal{P}}_{+}^{\uparrow}(s)$, we keep the same notation).

Let now $U$ be an irreducible, positive energy, massless, unitary representation $U$ of $\widetilde{\mathcal{P}}_{+}^{\uparrow}(s)$ with infinite spin $\kappa>0$. Then $U$ is associated as above by induction with an irreducible representation $V$ of the little group $\widetilde{E}(s-1)$ of radius $\kappa$. As in Proposition 4.3 we have to show that $\left.V \cdot \beta_{t}\right|_{\tilde{E}(s-1)}$ is a representation of radius $e^{-t} \kappa$.

Indeed, due to the relation (18), with $X \in \mathfrak{l i e}(E(2)) \subset \mathfrak{l i e}(E(s-1))$ we have

$$
\operatorname{spec}(i V(X))=\left[-e^{-t} \kappa, e^{-t} \kappa\right]
$$

so $U$ is not dilation covariant by the above comment.

An analogous discussion shows that finite helicity representations are dilation covariant.

The case $s=2$ is discussed here below.

Case $s=2$. In $2+1$ spacetime dimensions, the Lorentz group $\mathcal{L}_{+}(2)$ is isomorphic to $\operatorname{SL}(2, \mathbb{R}) /\{1,-1\}$. The little group of the point $q=(1,0,1)$ is $\mathbb{R}$, which is simply connected, and lifts uniquely to a one-parameter subgroup of the universal (infinite sheet) cover $\widetilde{\mathcal{L}}_{+}(2)$. The pre-image of the little group in $\mathcal{L}_{+}(2)$ is thus isomorphic to $\mathbb{R} \times \mathbb{Z}$, with $\mathbb{Z}$ the centre of $\widetilde{\mathcal{L}}_{+}(2)$.

The irreducible representations of the little group $\mathbb{R} \times \mathbb{Z}$ are thus one-dimensional, given by a pair $(\kappa, z)$ where $\kappa$ belongs to $\mathbb{R}$ (the dual of $\mathbb{R}$ ) and $z \in \mathbb{T}$ (the dual of $\mathbb{Z}$ ).

Denote by $U_{\kappa, z}$ the representation of $\widetilde{\mathcal{P}}_{+}^{\uparrow}(2)$ associated with the representation $(\kappa, z)$ of the little group. In analogy with the higher-dimensional case, we say that a unitary representation $U_{\kappa, z}$ of $\widetilde{\mathcal{P}}_{+}^{\uparrow}(2)$ has "infinite spin" if $\kappa \neq 0$. Yet, in this case, the name "infinite spin" does not refer to any infinite-dimensional representation.

Again, Eq. (18) holds, thus the representation $(\kappa, z)$ composed with the restriction of $\beta_{t}$ to the little group is equal to $\left(e^{-t} \kappa, z\right)$. It follows that $U_{\kappa, z}$ is dilation covariant iff $\kappa=0$.

We also notice that the conjugate representation of $(\kappa, z)$ is $(-\kappa, \bar{z})$, thus $U_{\kappa, z}$ extends to a (anti-)unitary representation of $\mathcal{P}_{+}(2)$, iff $\kappa=0$ and $z= \pm 1$. The other irreducible massless representations of $\mathcal{P}_{+}(2)$ are given by $U_{\kappa, z} \oplus U_{-\kappa, \bar{z}}$, with $\kappa \neq 0$ or $z \neq \pm 1$.

8.2. Twisted timelike locality. The second step consists of showing an analogous of Proposition 5.4 in any spacetime dimension $s \geq 2$.

We start with a unitary massless representation $U$ acting covariantly on a net on wedges $\mathcal{W} \ni W \longmapsto H(W) \subset \mathcal{H}$ s.t. assumptions 1-5 hold. Furthermore, suppose that for some double cone, the subspace $H(O)$, defined as in (13), is not trivial. In this setting the proof of Proposition 5.2 straightforwardly extends to every spacetime dimension.

Case s odd When the space dimension $s$ is odd, the Huygens principle holds and the proof of Proposition 5.4 easily extends in this case. 
Case s even, $s \geq 2$ In this case, timelike commutativity does not hold. Our results hold true, but Lemma 5.3, necessary to show that $H\left(V_{+}\right)$is separating, needs a variation.

As is well known, the Huygens principle is not satisfied in odd space dimensions, due to reverberations, yet we show here a version of this principle that holds if $s$ is even.

Let $f$ be a tempered distribution on $\mathbb{R}^{s+1}$; we define $\mathfrak{h}(f)$ by its Fourier transform

$$
\widehat{\mathfrak{h}(f)}(p)=-i \operatorname{sign}\left(p_{0}\right) \hat{f}(p),
$$

provided this expression is well defined. $\mathfrak{h}$ is the Hilbert transform with respect to the time variable, thus

$$
\mathfrak{h}(f)(x)=\frac{1}{\pi} \int_{-\infty}^{\infty} \frac{f\left(t, x_{1}, \ldots x_{s}\right)}{x_{0}-t} d t
$$

(integral in the principal value sense for a continuous function). Clearly, if $f_{1} \in$ $S\left(\mathbb{R}^{s+1}\right), f_{2} \in S^{\prime}\left(\mathbb{R}^{s+1}\right)$, the convolution product satisfies

$$
\mathfrak{h}\left(f_{1} * f_{2}\right)=\mathfrak{h}\left(f_{1}\right) * f_{2}=f_{1} * \mathfrak{h}\left(f_{2}\right)
$$

If $f$ is a function which is the boundary value of an analytic function on the tube

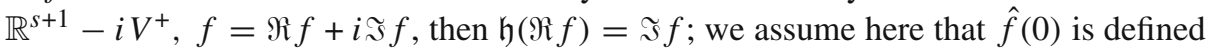
and equal to zero (to rule out the non-zero constants), namely $f$ has zero mean.

We are interested in the case $f$ is a solution of the wave equation $\square f=0$, then also $\square \mathfrak{h}(f)=0$. Let $\Delta_{+}$be the massless, scalar two-point function, namely the Fourier anti-transform of the Lorentz invariant measure on $\partial V_{+} \backslash\{0\}$. We have (up to a real proportionality constant), see e.g. [15],

$$
\Delta_{+}(x)=1 /|x|^{s-1} \text { if } \quad x^{2} \equiv x_{0}^{2}-x_{1}^{2}-\cdots x_{s}^{2} \neq 0,
$$

where $|x|=\sqrt{-x^{2}}$ (with opposite square root determination in $V_{ \pm}$) and

$$
\begin{aligned}
& \Delta_{+}(x) \text { real, } \quad \Delta_{+}(x)=\Delta_{+}(-x), \quad x \text { spacelike }\left(x^{2}<0\right) \\
& \Delta_{+}(x) \text { imaginary, } \Delta_{+}(x)=-\Delta_{+}(-x), \quad x \text { timelike }\left(x^{2}>0\right) \text {. }
\end{aligned}
$$

The commutator function

$$
\Delta_{0}(x)=\Delta_{+}(x)-\Delta_{+}(-x)
$$

vanishes for $x$ spacelike, while the function

$$
\Delta_{0}^{\prime}(x)=-i\left(\Delta_{+}(x)+\Delta_{+}(-x)\right)
$$

vanishes for $x$ timelike. Notice that we have

$$
\Delta_{0}^{\prime}=\mathfrak{h}\left(\Delta_{0}\right)
$$

Proposition 8.2. Let $f$ be a bounded continuous function on $\mathbb{R}^{s+1}$ with $\square f=0$, and $O$ a double cone. If $f(x)=0$ for $x$ in the spacelike complement of $O$, then $\mathfrak{h}(f)(x)=0$ for $x$ in the timelike complement of $O$. 
Proof. Let $h$ be a smooth function with $\operatorname{supp}(h) \subset O$. Then $f \equiv h * \Delta_{0}$ satisfies $\square f=0$ and $f(x)=0$ if $x \in O^{\prime}$. Moreover

$$
\mathfrak{h}(f)=\mathfrak{h}\left(h * \Delta_{0}\right)=h * \mathfrak{h}\left(\Delta_{0}\right)=h * \Delta_{0}^{\prime}
$$

vanishes on the timelike complement of $O$.

Now any smooth function $f$ with $\square f=0$ and $\operatorname{supp}(f) \subset O$ can be written $f=$ $h * \Delta_{0}$ as above, hence the proposition holds true for every smooth solution of the wave equation $f$. For a general continuous $f$, one can approximate as usual $f$ by $f * j_{\varepsilon}$ by a smooth approximate identity $j_{\varepsilon}$, and get the thesis because $\mathfrak{h}\left(f * j_{\varepsilon}\right)=\mathfrak{h}(f) * j_{\varepsilon}$.

We are now ready to prove the version of Lemma 5.3 in odd spacetime dimensions.

Lemma 8.3. Let $U$ be a massless, unitary representation of the double cover of the Poincaré group $\mathcal{P}_{+}^{\uparrow}(s)$, s even, $s \geq 2$, on a Hilbert space $\mathcal{H}$. Assume that $H$ is a twistedlocal, $U$-covariant net of standard subspaces of $\mathcal{H}$ on wedges. Let $\mathrm{O}_{1}, \mathrm{O}_{2} \in \mathcal{O}$ with $\mathrm{O}_{2}$ in the timelike complement of $O_{1}$, then

$$
H\left(O_{2}\right) \subset i Z H\left(O_{1}\right)^{\prime} .
$$

Proof. With $O_{r}$ and $f(x)=\langle\xi, U(x) Z \eta\rangle, \xi, \eta \in H\left(O_{r}\right)$ as in the proof of Lemma 5.3, we have $\square \mathfrak{s} f=0$ and $\mathfrak{s} f(x)=0$ if $x \in O_{2 r}^{\prime}$.

Thus, by Proposition 8.2, h( $(f(f))=-\mathfrak{R}(f)$ vanishes in the timelike complement of $\mathrm{O}_{2 r}$; but

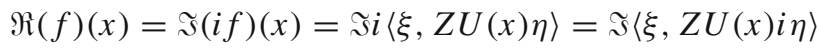

and we get the thesis.

We may now extend Proposition 5.4 in any spacetime dimension. Note that, in the following Proposition 8.4, the cyclicity assumption for $H(O)$ follows from $H(O) \neq\{0\}$ by Proposition 5.2.

Proposition 8.4. Let $U$ be a massless representation of $\widetilde{\mathcal{P}}_{+}^{\uparrow}$, acting covariantly on a net $H$ of standard subspaces of $\mathcal{H}$, satisfying $1-5$, on wedges on the $s+1$-dimensional Minkowski spacetime, with $s \geq 2$.

If $H(O)$ is cyclic for some double cone $O$, then $U$ is dilation covariant.

Moreover the dilation one-parameter unitary group $D$ can be chosen canonically, and $D(t) \in U\left(\widetilde{\mathcal{P}}_{+}^{\uparrow}\right)^{\prime \prime}, t \in \mathbb{R}$.

Proof. $H\left(V_{+}\right)$, the closed linear span of all spaces $H(O)$ with $O \subset V_{+}$, is a standard subspace of $\mathcal{H}$ by Lemma 8.3 , so, by positivity of the energy and Theorem 2.4 , the rescaled modular unitary group $D(t) \equiv \Delta_{H\left(V_{+}\right)}^{-i \frac{t}{2 \pi}}$ implements dilations on $U$-translations, and commutes with the Lorentz unitaries by Lemma 2.3. Namely $D$ implements the dilations on $U$.

Now, by Proposition 5.1, $U$ extends to an (anti)-unitary representation $\hat{U}$ of $\widetilde{\mathcal{P}}_{+}$on $\mathcal{H}, \hat{U}$ maps the reflection around the edge of $W$ to $J_{H(W)}$, and $H(W)=H_{\hat{U}}(W)$, the standard subspace associated by $\hat{U}$ with $W$.

Our choice of $D$ is canonical as it is given by modular unitaries. To show that $D(t) \in$ $U\left(\widetilde{\mathcal{P}}_{+}^{\uparrow}\right)^{\prime \prime}$, notice that this trivially holds if $U$ is irreducible. Recall now that finite helicity 
representations are dilation covariant. Assume first that $\hat{U}$ is an irreducible, finite nonzero helicity $h$ representation of $\widetilde{\mathcal{P}}_{+}$, then $\hat{U}$ restricts to $U=U_{h} \oplus U_{-h}$ on $\widetilde{\mathcal{P}}_{+}^{\uparrow}$, where $U_{h}$ is the helicity $h$ irreducible representation of $\widetilde{\mathcal{P}}_{+}^{\uparrow}$. There is a unitary implementation of dilations $T(s)$ which decomposes according to $U$. As $T(s) D(-s) \in U\left(\widetilde{\mathcal{P}}_{+}^{\uparrow}\right)^{\prime}$ and $U_{h}$ and $U_{-h}$ are disjoint, also $D(s)$ decomposes according to $U$, and $D(t) \in U\left(\widetilde{\mathcal{P}}_{+}^{\uparrow}\right)^{\prime \prime}$ holds.

In the general case with $U$ reducible, $U$ extends as above to a representation $\hat{U}$ of $\widetilde{\mathcal{P}}_{+}$, and so the net $H_{\hat{U}}$ disintegrates according to $\hat{U}$. In particular, $H\left(V_{+}\right)$and its modular unitaries disintegrate according to $\hat{U}$ and we have $D(t) \in U\left(\widetilde{\mathcal{P}}_{+}^{\uparrow}\right)^{\prime \prime}$ as stated.

We note that, by Lemma 2.2, if $s$ is even we have

$$
H\left(V_{+}\right)=i Z H\left(V_{-}\right)^{\prime} .
$$

In particular, if $H$ is local, we have twisted timelike duality $H\left(V_{+}\right)=i H\left(V_{-}\right)^{\prime}$, and if $H$ is purely Femi-local $(Z=-i)$ we have timelike duality $H\left(V_{+}\right)=H\left(V_{-}\right)^{\prime}$.

8.3. General result. We indicate in this section the modifications that are necessary to extend our results in any spacetime dimension $s+1 \geq 3$.

Let $U$ be a unitary, positive energy representation of $\widetilde{\mathcal{P}}_{+}^{\uparrow}(s)$ on a Hilbert space $\mathcal{H}$. We assume here that a $2 \pi$-rotation in space gives a selfadjoint operator $\Gamma \equiv U(2 \pi)$, i.e., the eigenvalues of $\Gamma$ are \pm 1 . In other words $U$ is a representation of the double cover of $\mathcal{P}_{+}^{\uparrow}(s)$ which coincides with the universal cover $\widetilde{\mathcal{P}}_{+}^{\uparrow}(s)$ if $s>2$; and $\Gamma$ is the image under $U$ of the non-trivial element in the centre of the double cover.

A $U$-covariant (twisted-local) net of standard subspaces $H$ is defined as a map

$$
\mathcal{W} \ni W \longmapsto H(W) \subset \mathcal{H}
$$

as in Sect. 5.

Note that the proof Proposition 5.2 does not use the twisted locality property, and is valid also here. We have:

Theorem 8.5. Let $U$ be a unitary, positive energy representation of the cover of the Poincaré group $\widetilde{\mathcal{P}}_{+}^{\uparrow}(s)$, acting covariantly on a net $H$ of standard subspaces of $\mathcal{H}$ on wedges satisfying $1-5$ as above, $s \geq 2$.

(a) If $H(O)$ is cyclic for some double cone $O$, then $U$ does not contain an infinite spin sub-representation (namely there is no infinite spin fibre in the irreducible direct integral decomposition).

(b) If $U$ is irreducible and $H(O) \neq\{0\}$ for some double cone $O$, then $U$ is not massless with infinite spin.

(c) If $U$ extends to an (anti-)unitary, irreducible representation $\hat{U}$ of $\widetilde{\mathcal{P}}_{+}$on $\mathcal{H}$ and $H(O) \neq\{0\}$ for some double cone $O$, then $U$ does not contain an infinite spin sub-representation.

Proof. (b) follows from (a) by Proposition 5.2, so we prove the statement (a).

By restricting to the massless component, we may assume that $U$ is massless. By Proposition 8.4, $U$ is dilation covariant; $U=\int_{X}^{\oplus} U_{\lambda} d \mu(\lambda)$ is the irreducible direct integral decomposition of $U$, by Proposition 8.4 the dilation unitary group $D$ decomposes 
accordingly, $D=\int_{X}^{\oplus} D_{\lambda} d \mu(\lambda)$, so $U_{\lambda}$ is dilation covariant for $\mu$-almost all $\lambda$. Thus $U_{\lambda}$ has not infinite spin by Proposition 8.1.

(c) Either $U$ is irreducible, and we apply (b), or $U$ is the direct sum of two irreducible, inequivalent representations of $\widetilde{\mathcal{P}}_{+}^{\uparrow}, U=U_{1} \oplus U_{2}$ on $\mathcal{H}_{1} \oplus \mathcal{H}_{2}$. In this case, let $\mathcal{K} \subset \mathcal{H}$ be the complex Hilbert subspaces generated by $H$. Then $\mathcal{K}$ is $U$-invariant. If $\mathcal{K}=\mathcal{H}$ we apply (a). Otherwise $\left.U\right|_{\mathcal{K}}=U_{1}$ (or $\left.U\right|_{\mathcal{K}}=U_{2}$ ). Then $H(O)$ is cyclic on $\mathcal{K}$ for some double cone as in Proposition 5.2, so $U_{1}$ extends to an (anti-)unitary representation of $\widetilde{\mathcal{P}}_{+}$ on $\mathcal{H}_{1}$ [11]; thus $\hat{U}$ is easily seen to be reducible, contrary to our assumption. Therefore $\mathcal{K}=\mathcal{H}$, and the conclusion follows from (a).

\section{Quantum Field Theory: Nets of von Neumann Algebras}

In this section the Minkowski spacetime dimension is $s \geq 2$.

Given a positive energy (anti-)unitary representation of the proper Poincaré group $\mathcal{P}_{+}$ on a Hilbert space $\mathcal{H}$, the paper [4] provides a canonical construction of a $U$-covariant local net of standard subspaces of $\mathcal{H}$ on wedges with the properties 1-5. Similarly, this construction gives a twisted-local canonical $U$ covariant net if one considers a representation $U$ of the the universal cover $\widetilde{\mathcal{P}}_{+}$. The above Theorems $6.1,8.5$ apply to this net, hence to the net of von Neumann algebras obtained via second quantisation on the Bose/Fermi Fock space, depending on $U(2 \pi)= \pm 1$.

A twisted-local, $U$-covariant net of von Neumann algebras on wedges $\mathcal{F}$ is an isotonous map

$$
W \longmapsto \mathcal{F}(W)
$$

that associates a von Neumann algebra $\mathcal{F}(W)$ on a fixed Hilbert space $\mathcal{H}$ with every $W \in \mathcal{W}$, with the following properties:

- Poincaré covariance: $U(g) \mathcal{F}(W) U(g)^{*}=\mathcal{F}(g W), g \in \widetilde{\mathcal{P}}_{+}^{\uparrow}$;

- Vacuum with Reeh-Schlieder property: there exists a unique (up to a phase) $U$ invariant vector $\Omega \in \mathcal{H}$ and $\mathcal{F}(W)$ is cyclic on $\Omega$ for all $W \in \mathcal{W}$;

- Bisognano-Wichmann property:

$$
\Delta_{W}^{i t}=U\left(\Lambda_{W}(-2 \pi t)\right), \quad W \in \mathcal{W},
$$

where $\Delta_{W}$ is the modular operator of $(\mathcal{F}(W), \Omega)$;

- Twisted locality: For every wedge $W \in \mathcal{W}$ we have

$$
Z \mathcal{F}\left(W^{\prime}\right) Z^{*} \subset \mathcal{F}(W)^{\prime}
$$

where $Z$ is unitary and $Z=\frac{1+i \Gamma}{1+i}, \Gamma=U(2 \pi)$ as above.

Due to twisted locality, $\Omega$ is indeed also separating for each $\mathcal{F}(W)$, so the modular operators $\Delta_{W}$ are defined.

Given $\mathcal{F}$ as above, we define the von Neumann algebra associated with the region $O$ as

$$
\mathcal{F}(O) \equiv \bigcap_{\mathcal{W} \ni W \supset O} \mathcal{F}(W)
$$

A twisted-local, $U$ covariant net $O \longmapsto \mathcal{F}(O)$ on double cones is analogously defined, by requiring the $U$-covariance and the cyclicity of the algebras $\mathcal{F}(O)$. Then $\mathcal{F}(W)$ is defined by additivity and $W \longmapsto \mathcal{F}(W)$ is a twisted-local, $U$-covariant net on wedges. 
The von Neumann algebras $\mathcal{F}(O)$ defined by (21) are, in general, larger than the original $\mathcal{F}(O)$ (they define the dual net).

The free Bose (resp. Fermi) field net $\mathcal{F}_{ \pm}$is defined by second quantization on the symmetric/anti-symmetric Fock space $\mathrm{F}_{ \pm}(\mathcal{H})$ as

$$
\mathcal{F}_{ \pm}(W) \equiv R_{ \pm}(H(W)), \quad W \in \mathcal{W}
$$

where $H=H_{U}$ is the canonical net of standard subspaces of the one-particle Hilbert space $\mathcal{H}$ associated with the unitary representation $U$ of the cover of Poincare group with $U(2 \pi)= \pm \mathbf{1}$, and $R_{ \pm}(H(W))$ are defined as follows.

With $\mathcal{H}$ a Hilbert space and $H \subset \mathcal{H}$ a real linear subspace, $R_{ \pm}(H)$ is the von Neumann algebra on $\mathrm{F}_{ \pm}(\mathcal{H})$ generated by the CCR/CAR operators:

$$
R_{+}(H) \equiv\{\mathrm{w}(\xi): \xi \in H\}^{\prime \prime}, \quad R_{-}(H) \equiv\{\Psi(\xi): \xi \in H\}^{\prime \prime},
$$

with $\mathrm{w}(\xi)$ the Weyl unitaries on $\mathrm{F}_{+}(\mathcal{H})$ and $\Psi(\xi)$ the Fermi field operators on $\mathrm{F}_{-}(\mathcal{H})$.

Note that, by continuity,

$$
R_{ \pm}(H)=R_{ \pm}(\bar{H}) .
$$

Moreover the vacuum vector $\Omega$ is cyclic (resp. separating) for $R_{ \pm}(H)$ iff $\bar{H}$ is cyclic (resp. separating).

If $H$ is standard, we denote by $S_{H}^{ \pm}, J_{H}^{ \pm}, \Delta_{H}^{ \pm}$the Tomita operators associated with $\left(R_{ \pm}(H), \Omega\right)$, and by $\Gamma_{ \pm}(T)$ the Bose/Fermi second quantization of a one-particle operator $T$ on $\mathcal{H}$, defined by tensor products on $\mathrm{F}_{ \pm}(\mathcal{H})$.

This assignment (22) respects the lattice structure, as originally proven in [1] (Bose case) and [10] (Fermi case). The modular operators were computed in [9,10,21]. For convenience, we state these properties in the following proposition with a sketch of proof.

Proposition 9.1. Let $H$ and $H_{a}$ be closed, real linear subspaces of $\mathcal{H}$. We have

$\left(\mathrm{a}_{+}\right) S_{H}^{+}=\Gamma_{+}\left(S_{H}\right), J_{H}^{+}=\Gamma_{+}\left(J_{H}\right), \Delta_{H}^{+}=\Gamma_{+}\left(\Delta_{H}\right)$,

(a $) S_{H}^{-}=Z \Gamma_{-}\left(i S_{H}\right), J_{H}^{-}=Z \Gamma_{-}\left(i J_{H}\right), \Delta_{H}^{-}=\Gamma_{-}\left(\Delta_{H}\right)$,

(b) $R_{+}(H)^{\prime}=R_{+}\left(H^{\prime}\right)$ and $R_{-}(H)^{\prime}=Z R_{-}\left(i H^{\prime}\right) Z^{*}$,

(c) $R_{ \pm}\left(\sum_{a} H_{a}\right)=\bigvee_{a} R_{ \pm}\left(H_{a}\right)$

(d) $R_{ \pm}\left(\cap_{a} H_{a}\right)=\bigcap_{a} R_{ \pm}\left(H_{a}\right)$

where $\bigvee$ denotes the von Neumann algebra generated, $Z=\mathbf{1}$ (resp. $Z=-i$ ) on the $n$-particle subspace, $n$ even (resp. odd), and $H$ is standard in $\left(a_{ \pm}\right)$.

Proof. $\left(\mathrm{a}_{ \pm}\right) S_{H}^{+}=\Gamma_{+}\left(S_{H}\right)$ due to the relation $S_{H}^{+} \mathrm{w}(\xi) \Omega=\mathrm{w}(-\xi) \Omega$ (see [21]), while $S_{H}^{-}=Z \Gamma_{-}\left(i S_{H}\right)$ due to the relation $S_{H}^{-} \Psi\left(\xi_{1}\right) \Psi\left(\xi_{2}\right) \ldots \Psi\left(\xi_{n}\right) \Omega=\Psi\left(\xi_{n}\right) \ldots \Psi\left(\xi_{2}\right) \Psi$ $\left(\xi_{1}\right) \Omega, \xi \in H$, with $\Omega$ the Fock vacuum vector (see [10]). By the uniqueness of the polar decomposition, we then have $J_{H}^{+}=\Gamma_{+}\left(J_{H}\right), J_{H}^{-}=Z \Gamma_{-}\left(i J_{H}\right)$ and $\Delta_{H}^{ \pm}=\Gamma_{ \pm}\left(\Delta_{H}\right)$.

(b) Assume first that $H$ is standard. By (a) we have

$$
\begin{gathered}
R_{+}(H)^{\prime}=J_{H}^{+} R_{+}(H) J_{H}^{+}=R_{+}\left(J_{H} H\right)=R_{+}\left(H^{\prime}\right), \\
R_{-}(H)^{\prime}=J_{H}^{-} R_{-}(H) J_{H}^{-}=Z R_{-}\left(i J_{H} H\right) Z^{*}=Z R_{-}\left(i H^{\prime}\right) Z^{*} .
\end{gathered}
$$

Now (b) trivially holds for $H=\mathcal{H}$ or $H=\{0\}$ too. To prove (b) for a general closed real linear subspace $H$ of $\mathcal{H}$, we may decompose $\mathcal{H}$ in the direct sum $\mathcal{H}=\mathcal{H}_{-1} \oplus \mathcal{H}_{0} \oplus \mathcal{H}_{1}$, where $\mathcal{H}_{1} \equiv H^{\perp}$ and $\mathcal{H}_{-1} \equiv H \cap i H$. Then $H$ decomposes as $H=H_{-1} \oplus H_{0} \oplus H_{1}$ 
with $H_{-1}=\mathcal{H}_{-1}, H_{1}=\{0\}$ and $H_{0}$ a standard subspace of $\mathcal{H}_{0}$, and the statement follows at once.

(c) is an immediate consequence of the Weyl relations $\mathrm{w}(\xi+\eta)=e^{-\Im\langle\xi, \eta\rangle} \mathrm{w}(\xi) \mathrm{w}(\eta)$ (Bose case), the real linearity of $\Psi$ (Fermi case), and (a).

(d) now follows easily from (b) and (c).

We state now the following consequence of Theorems $6.1,8.5$ for free fields.

Corollary 9.2. Let $\mathcal{F}_{ \pm}$be the free Bose/Fermi field net of von Neumann algebras on wedges associated with a positive energy, infinite spin, irreducible unitary Bose/Fermi representation of $\widetilde{\mathcal{P}}_{+}^{\uparrow}[4]$.

Then $\mathcal{F}(C)$ is cyclic on the vacuum vector if $C$ is a spacelike cone, but $\mathcal{F}(O)=\mathbb{C} \cdot \mathbf{1}$ if $O$ is any bounded spacetime region.

Proof. Immediate by Theorem 6.1, the results in [4], and the fact (Theorem 9.1) that the intersection of closed real linear spaces of the one-particle Hilbert space corresponds to the intersection of the associated von Neumann algebras on the Fock space:

$$
\bigcap_{W \supset X} \mathcal{F}_{ \pm}(W) \equiv \bigcap_{W \supset X} R_{ \pm}(H(W))=R_{ \pm}\left(\bigcap_{W \supset X} H(W)\right)
$$

for $X=C$ a cone, resp. $X=O$ a double cone.

The following theorem shows why infinite spin representations do not occur in a theory of local observables.

We shall say that a unitary representation $U$ of $\widetilde{\mathcal{P}}_{+}^{\uparrow}$ has infinite spin if $U$ is a direct integral of irreducible, infinite spin representations. Thus $U$ does not not contain an infinite spin sub-representation iff no infinite spin representation appears in the irreducible direct integral decomposition of $U$ (up to a measure zero set).

Theorem 9.3. Let $\mathcal{F}$ be a twisted-local net of von Neumann algebras $\mathcal{F}(O)$ on double cones on a Hilbert space $\mathcal{H}$, covariant w.r.t. a unitary representation $U$ of $\widetilde{\mathcal{P}}_{+}^{\uparrow}$ with vacuum vector $\Omega \in \mathcal{H}$. As above, we assume the double cone Reeh-Schlieder property and the Bisognano-Wichmann property.

Then $U$ does not contain an infinite spin sub-representation.

Proof. For every wedge $W \in \mathcal{W}$ we set

$$
H(W)=\overline{\mathcal{F}(W)_{\text {s.a. }} \Omega},
$$

where $\mathcal{F}(W)_{\text {s.a. }}$ is the selfadjoint part of $\mathcal{F}(W)$. By assumptions, $H: W \longmapsto H(W)$ is then a twisted-local, $U$-covariant net of standard subspaces of $\mathcal{H}$ satisfying Properties $1-5$.

With $O$ a double cone, we have that

$$
H(O) \equiv \bigcap_{\mathcal{W} \ni W \supset O} H(W) \supset \mathcal{F}(O)_{\text {s.a. }} \Omega
$$

is cyclic.

We thus infer from Theorem 8.5 (a) that $U$ does not contain an infinite spin subrepresentation. 
We now start with a local net $\mathcal{A}$ of von Neumann algebras on double cones, with the double cone Reeh-Schlieder property and the Bisognano-Wichmann property. Let

$$
\mathfrak{A} \equiv \overline{\bigcup_{O \in \mathcal{O}} \mathcal{A}(O)}
$$

(norm closure) be the quasi-observable $C^{*}$-algebra. We shall say that a representation $\pi$ of $\mathfrak{A}$ is cone localizable if, for every spacelike cone $C,\left.\pi\right|_{\mathfrak{A}\left(C^{\prime}\right)}$ is unitarily equivalent to id $\left.\right|_{\mathfrak{A}\left(C^{\prime}\right)}$, where $\mathfrak{A}(C)$ is the $C^{*}$-algebra generated by $\mathcal{A}(O)$ as $O$ runs in the double cones contained in $C$. Similarly $\pi$ is double cone localizable if $\left.\left.\pi\right|_{\mathfrak{A}\left(O^{\prime}\right)} \simeq \mathrm{id}\right|_{\mathfrak{A}\left(O^{\prime}\right)}$, for all double cones $O$.

A Doplicher-Haag-Roberts (DHR) (resp. a Buchholz-Fredenhagen) representation $[5,7]$ is a Poincaré covariant representation with positive energy, which is double cone (resp. cone) localizable (Poincaré covariance with positive energy follows by general assumptions [11]).

Theorem 9.4. Let $\pi$ be a DHR representation of $\mathfrak{A}$ with finite statistics [7]. Then the unitary representation $U_{\pi}$ of $\widetilde{\mathcal{P}}_{+}^{\uparrow}$ in the representation $\pi$ does not contain infinite spin sub-representations.

Proof. By considering the dual net, we can assume Haag duality for double cones. We consider the Doplicher-Roberts twisted-local field net $\mathcal{F}$. We have $\mathcal{A}(O) \subset \mathcal{F}(O)$ and the restriction of the vacuum representation of $\mathcal{F}$ to $\mathcal{A}$ is the direct sum (with multiplicity) of all DHR representations of $\mathcal{A}$ with finite statistics.

The representation $U_{\mathcal{F}}$ of $\widetilde{\mathcal{P}}_{+}^{\uparrow}$ restricts accordingly to the representations of $\mathcal{A}$. Thus we have to show that $U_{\mathcal{F}}$ does not contain an infinite spin sub-representation. This will follow from Theorem 9.3 once we show the Bisognano-Wichmann property. Now the Bisognano-Wichmann property for $\mathcal{F}$ is a consequence of the Bisognano-Wichmann property for $\mathcal{A}$ as one can identify the Connes-Radon-Nikodym cocycles, see [16,23].

As a consequence, let $\pi$ be a Poincaré covariant representation of $\mathfrak{A}$. If $\pi$ contains infinite spin particles (i.e., $U_{\pi}$ contains an infinite spin sub-representation) then:

$$
\pi \text { is localizable in a double cone } \Longrightarrow \pi \text { has infinite statistics. }
$$

This indicates an intimate relation among infinite spin, infinite statistics and localization in infinitely extended regions.

\section{Final Comments}

10.1. Field algebra structure. We now describe the field algebra structure that we obtain starting from the observable algebra and adding all charges with finite statistics, including the ones with infinite spin (space dimension $s>2$ ).

Let $\mathcal{A}$ be as a above a local net with the double cone Reeh-Schlieder property and the Bisognano-Wichmann property. Let $\mathcal{T}$ be the family of all irreducible representations, up to unitary equivalence (sectors), of $\mathfrak{A}$ of Buchholz-Fredenhagen type with finite statistics.

The Doplicher-Roberts construction [8] yields a field net $\mathcal{F}$ of von Neumann algebras on a larger Hilbert space with $\mathcal{F}(C) \supset \mathcal{A}(C)$ for every cone $C$, and the identity representation of $\mathcal{A}$ on the Hilbert space of $\mathcal{F}$ decomposes into the direct sum of elements of $\mathcal{T}$, with multiplicity. 
By the spin-statistics theorem [12], $\mathcal{F}$ is a twisted-local net. If infinite spin sectors exist, then by Theorem $9.3 \mathcal{F}(O)$ cannot be cyclic on the vacuum vector if $O$ is a bounded region. If one restricts $\mathcal{F}$ to the cyclic Hilbert space generated by $\mathcal{F}(O)$, one gets the field algebra associated with DHR charges. We discuss a physical interpretation of this structure in the outlook.

We mention also that, in two space dimensions, cone localizable representations may have braid group statistics. If we consider only those ones with Bose or Fermi statistics, then the above field algebra description still holds (the spin-statistics theorem in $2+1$ dimensions is treated in [22]). However, with general statistics, no field algebra exists that describes an analogue of the above picture.

10.2. de Sitter spacetime. If $\mathcal{A}$ is a local net on spacelike cones of the Minkowski spacetime $\mathbb{R}^{s+1}$, one can associate a local net $\mathcal{B}$ on double cones of the $s$-dimensional de Sitter spacetime $d S_{S}$ (and similarly in the twisted-local case). As usual, one views $d S_{S}$ as an hyperboloid of $\mathbb{R}^{s+1}$, which is the manifold of spacelike directions of Minkowski spacetime. With $E$ any region of $d S_{s}$, one sets $\mathcal{B}(E) \equiv \mathcal{A}\left(C_{E}\right)$, where $C_{E} \subset \mathbb{R}^{s+1}$ is the spacelike cone with apex in the origin spanned by $E$.

This construction has been made in [4]. In particular, in the free field case (finite or infinite spin), one gets the canonical modular construction on $d S_{s}$ associated with the restriction of Poincaré unitary representation to the Lorentz subgroup.

We emphasize here that the de Sitter picture is natural in the presence of infinite spin particles. These particles have no bounded spacetime localization on the Minkowski spacetime, yet they are localized in bounded spacetime regions of the de Sitter spacetime.

10.3. The role of the Bisognano-Wichmann property. In this paper, we rely on the Bisognano-Wichmann property as a first principle (cf. [11,12]), so we briefly comment here on its roots.

The Bisognano-Wichmann property implies the positivity of the energy (see [4]), and is slightly stronger than that; it reflects the stability of the vacuum state. It always holds in a Wightman theory [2], including string localized fields [27]. In the local algebra framework one can find a counter-example (see Sect.7), which has however a pathological nature (with continuous degeneracy) and is built on the non-uniqueness of the covariance unitary representation of the Poincaré group: if one chooses the wrong (noncanonical) representation, one obviously violates the Bisognano-Wichmann property. So we may expect the latter to always hold when the Poincaré representation is unique, say by assuming the split property.

In a massive theory, the Bisognano-Wichmann property can be derived by asymptotic completeness [26]. It always holds in the conformal case. It is equivalent to a subexponential growth estimate on the energy density levels of localized states for the Rindler Hamiltonian, namely $\left(\xi, e^{-2 \pi K} \xi\right)<\infty$ for all vector states $\xi$ localized in a given cone $C$ contained in a wedge $W$, with $K$ the generator of the unitary one-parameter group of boosts associated with $W$ [13].

A further argument for the Bisognano-Wichmann property is its mentioned equivalence with the Hawking-Unruh effect for Rindler black holes [31] (the Hawking temperature is the KMS temperature). An illustration of this fact goes beyond the purpose of this paper and we refer to the book [14] for more insight on this point and related aspects. 


\section{Outlook}

Infinite spin particle states cannot be localized in a bounded spacetime region. This corresponds to the fact that no local observables exist that can generate these states from the vacuum. These results, obtained in the present paper in the Operator Algebraic intrinsic setup [4], extend the no-go theorem on local fields with infinite spin obtained previously in the Wightman setting [36]. The string-localized free fields constructed in [27], which correspond to and generate the von Neumann algebras in [4], cannot thus be compactly localized.

As described in Sect. 10.1 our results provide the following picture in a theory of local observables.

A quantum field theory on a Hilbert space including infinite spin states is described by a net $W \longmapsto \mathcal{F}(W)$ of von Neumann algebras for wedge regions, and the vacuum vector is cyclic for the von Neumann algebras for spacelike cones (defined by intersections of wedge algebras), and has the Bisognano-Wichmann property. The algebras for double cone regions are non-trivial, forming a covariant subnet $O \longmapsto \mathcal{A}(O)$, but the vacuum is not cyclic for $\mathcal{A}(O)$. The full Hilbert space therefore splits into representations of $\mathcal{A}$, with the infinite spin states absent from the vacuum representation. The representations containing the infinite spin states are massless sectors of the Buchholz-Fredenhagen type, i.e., localized in spacelike cones, and the net $\mathcal{F}$ serves as a field algebra for these sectors. This picture complies with the scenario proposed by Schroer [30] with a hindsight on "dark matter".

One may reasonably expect that $\mathcal{A}$ contains local generators of Poincaré transformations, i.e., a stress-energy tensor subnet (which could couple to gravity). As infinite spin states are localized in spacelike cones, their Lorentz transforms will be localized in different cones. Thus, the obstruction against infinite spin states to be present in the vacuum representation is necessary because they cannot be Lorentz transformed by local generators. But the representatives of local generators in a cone-localized representation may well Lorentz transform infinite spin states present in these sectors.

By our result (Corollary 9.2), if $\mathcal{F}$ is the free field net associated with an infinite spin representation, then the subnet $\mathcal{A}$ would be trivial. Hence, the above scenario necessarily requires a self-interaction of some unknown sort. It is an exciting challenge to describe such an interaction, and the possible interaction of infinite spin fields with "ordinary matter" fields.

Acknowledgements. We thank S. Carpi, D. Guido, G. Morsella, and J. Yngvason for useful discussions.

Open Access This article is distributed under the terms of the Creative Commons Attribution 4.0 International License (http://creativecommons.org/licenses/by/4.0/), which permits unrestricted use, distribution, and reproduction in any medium, provided you give appropriate credit to the original author(s) and the source, provide a link to the Creative Commons license, and indicate if changes were made.

\section{References}

1. Araki, H.: A lattice of von Neumann algebras associated with the quantum theory of a free Bose field. J. Math. Phys. 4, 1343-1362 (1963)

2. Bisognano, J.J., Wichmann, E.H.: On the duality condition for quantum fields. J. Math. Phys. 17, 303321 (1976)

3. Borchers, H.-J.: The CPT theorem in two-dimensional theories of local observables. Commun. Math. Phys. 143, 315-332 (1992)

4. Brunetti, R., Guido, D., Longo, R.: Modular localization and Wigner particles. Rev. Math. Phys. 14(7 \& 8), 759-786 (2002) 
5. Buchholz, D., Fredenhagen, K.: Locality and structure of particle states. Commun. Math. Phys. 84, 154 (1982)

6. Buchholz, D., Porrmann, M., Stein, U.: Dirac versus Wigner: towards a universal particle concept in local quantum field theory. Phys. Lett. B 267, 377-381 (1991)

7. Doplicher, S., Haag, R., Roberts, J.E.: Local observables and particle statistics. I. Commun. Math. Phys. 23, 199-230 (1971)

8. Doplicher, S., Roberts, J.E.: Why there is a field algebra with a compact gauge group describing the superselection structure in particle physics. Commun. Math. Phys. 131, 51-107 (1990)

9. Eckmann, J.P., Osterwalder, K.: An application of Tomita's theory of modular Hilbert algebras: duality for free Bose fields. J. Funct. Anal. 13, 1-12 (1973)

10. Foit, J.J.: Abstract twisted duality for quantum free Fermi fields. Publ. RIMS Kyoto Univ. 19, 729$74(1983)$

11. Guido, D., Longo, R.: Relativistic invariance and charge conjugation in quantum field theory. Commun. Math. Phys. 148, 521-551 (1992)

12. Guido, D., Longo, R.: An algebraic spin and statistics theorem. Commun. Math. Phys. 172, 517-533 (1995)

13. Guido, D., Longo, R.: Natural energy bounds in quantum thermodynamics. Commun. Math. Phys. 218, 513-536 (2001)

14. Haag, R.: Local Quantum Physics-Fields, Particles, Algebras, 2nd edn. Springer, New York (1996)

15. Hislop, P.D., Longo, R.: Modular structure of the local algebras associated with the free massless scalar field theory. Commun. Math. Phys. 84, 71-85 (1982)

16. Isola, T.: Modular structure of the crossed product by a compact group dual. J. Oper. Theory 33, 3-31 (1995)

17. Iverson, G.J., Mack, G.: Quantum fields and interaction of massless particles: the continuous spin case. Ann. Phys. 64, 211-253 (1971)

18. Köhler, Ch.: On the localization properties of quantum fields with zero mass and infinite spin. Ph.D. thesis, Vienna (2015)

19. Lawson, H.B. Jr., Michelsohn, M.L.: Spin Geometry. Princeton University Press, Princeton (1989)

20. Lechner, G., Longo, R.: Localization in nets of standard spaces. Commun. Math. Phys. 336, 27-61 (2015)

21. Leyland, P., Roberts, J.E., Testard, D.: Duality for quantum free fields. Unpublished manuscript, Marseille (1978)

22. Longo, R.: On the spin-statistics relation for topological charges. In: Doplicher, S. et al. (eds.) Operator Algebras and Quantum Field Theory (Rome, 1996), pp. 661-669. Int. Press, Cambridge, MA (1997)

23. Longo, R.: An analogue of the Kac-Wakimoto formula and black hole conditional entropy. Commun. Math. Phys. 186, 451-479 (1997)

24. Longo, R.: Lectures on Conformal Nets. Preliminary lecture notes that are available at http://www.mat. uniroma2.it/ longo/Lecture_Notes.html

25. Longo, R.: Real Hilbert subspaces, modular theory, $\operatorname{SL}(2, \mathbb{R})$ and CFT. In: Von Neumann algebras in Sibiu, pp. 33-91, Theta Ser. Adv. Math., 10, Theta, Bucharest (2008)

26. Mund, J.: The Bisognano-Wichmann theorem for massive theories. Ann. Henri Poincaré 2, 907$926(2001)$

27. Mund, J., Schroer, B., Yngvason, J.: String-localized quantum fields from Wigner representations. Phys. Lett. B 596, 156-162 (2004)

28. Oksak, A.I., Todorov, I.T.: Invalidity of TCP theorem for infinite-component fields. Commun. Math. Phys. 11, 125-130 (1968)

29. Rieffel, M.A., Van Daele, A.: A bounded operator approach to Tomita-Takesaki theory. Pac. J. Math. 69, 187-221 (1977)

30. Schroer, B.: Manuscripts on infinite spin and dark matter. arXiv:0802.2098v3, arXiv:0802.2098v4, arXiv:1306.3876v5

31. Sewell, G.L.: Quantum fields on manifolds: PCT and gravitationally induced thermal states. Ann. Phys. 141, 201-224 (1982)

32. Streater, R.F., Wightman, A.S.: PCT, Spin and Statistics, and All that, 2nd edn. Addison-Wesley Publishing Company, Advanced Book Program, Redwood City (1989)

33. Takesaki, M.: Theory of Operator Algebras, I \& II. Springer-Verlag, New York-Heidelberg (2002, 2003)

34. Wigner, E.P.: On unitary representations of the inhomogeneous Lorentz group. Ann. Math. 40, 149204 (1939)

35. Yngvason, J.: Zur Existenz von Teilchen mit Masse 0 und unendlichem Spin in der Quantenfeldheorie. Diploma thesis, Göttingen (1969)

36. Yngvason, J.: Zero-mass infinite spin representations of the Poincaré group and quantum field theory. Commun. Math. Phys. 18, 195-203 (1970)

37. Zimmer, R.J.: Ergodic Theory of Semisimple Lie Groups. Birkhäuser, Boston-Basel-Stuttgart (1984) 